\title{
Chemical bonds of two vortex species with a generalized Josephson term and arbitrary charges
}

\author{
Chandrasekhar Chatterjee, ${ }^{a}$ Sven Bjarke Gudnason ${ }^{b}$ and Muneto Nitta ${ }^{a}$ \\ ${ }^{a}$ Department of Physics, and Research and Education Center for Natural Sciences, Keio University, \\ Hiyoshi 4-1-1, Yokohama, Kanagawa 223-8521, Japan \\ ${ }^{b}$ Institute of Contemporary Mathematics, School of Mathematics and Statistics, Henan University, \\ Kaifeng, Henan 475004, P.R. China \\ E-mail: chatterjee@keio.jp, gudnason@henu.edu.cn, \\ nitta@phys-h.keio.ac.jp
}

Abstract: We consider the Abelian-Higgs model with two complex scalar fields and arbitrary positive integer charges with the addition of a higher-order generalization of the Josephson term. The theory possesses vortices of both local and global variants. The only finite-energy configurations are shown to be the local vortices for which a certain combination of vortex numbers and electric charges - called the global vortex number - vanishes. The local vortices have rational fractional magnetic flux, as opposed to the global counterparts that can have an arbitrary fractional flux. The global vortices have angular domain walls, which we find good analytic approximate solutions for. Finally, we find a full classification of the minimal local vortices as well as a few nonminimal networks of vortices, using numerical methods.

KEywords: Solitons Monopoles and Instantons, Field Theories in Lower Dimensions

ARXiv EPrint: 1912.02685 


\section{Contents}

1 Introduction 1

2 The model, vortex asymptotics and angular domain walls 4

2.1 Symmetries 4

2.2 The vacuum 6

2.3 Vortex Ansatz 6

2.3.1 $\gamma=0 \quad 6$

2.3.2 $\gamma \neq 0$ : angular domain walls $\quad 8$

$\begin{array}{llr}2.4 & \text { Finite energy vortices } & 10\end{array}$

$\begin{array}{lll}2.4 .1 & \gamma=0 & 10\end{array}$

2.4.2 $\gamma \neq 0$ : angular domain walls 11

3 Numerical results $\quad 12$

3.1 Numerical method 12

$\begin{array}{ll}3.2 \text { Visualization } & 13\end{array}$

$\begin{array}{lll}3.3 & \text { An intuitive explanation } & 13\end{array}$

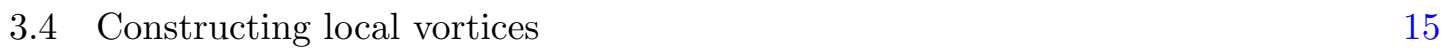

$\begin{array}{ll}3.5 & \text { Minimal solutions } \\ \end{array}$

3.5.1 $Q_{1}=Q_{2}=Q$ : the normal vortex $\quad 16$

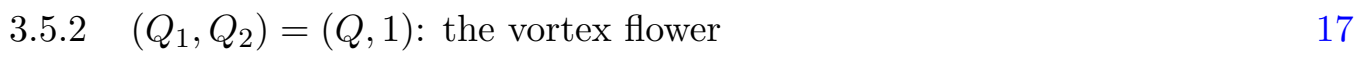

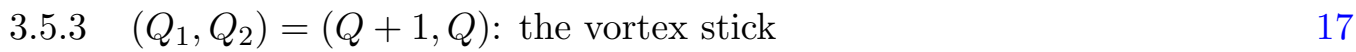

3.5.4 $\left(Q_{1}, Q_{2}\right)=(2 Q, Q):$ the vortex pill 18

$\begin{array}{ll}3.5 .5 & \left(Q_{1}, Q_{2}\right)=(4,3): \text { the extended flower }\end{array}$

$\begin{array}{lll}3.6 & \text { Nonminimal solutions } & 20\end{array}$

$\begin{array}{ll}3.7 & \text { Varying the VEVs }\end{array}$

$\begin{array}{lll}3.8 & \text { Global vortices } & 29\end{array}$

4 Conclusion and discussion $\quad 31$

\section{Introduction}

A type-II superconductor expels an applied magnetic field due to the Meissner effect, such that it flows around the superconducting material - with the exception of formation of magnetic vortices, called Abrikosov-Nielsen-Olesen (ANO) vortices [1, 2]. The latter can penetrate the superconductor in the direction of the externally applied magnetic field if the magnetic field is not too large and not too small. ANO vortices are elegantly described in the Ginzburg-Landau (GL) effective theory of superconductivity. The static vortices are independent of the theory being relativistic or nonrelativistic. $n$ vortices are described 
by $n$ zeroes in the order parameter of the theory, which is a complex scalar field, and has additionally a phase that winds $n$ times at spatial infinity (or at any radius far from the centers of the $n$ zeroes), see e.g. the text book [3].

The order parameter of a superconductor in the GL effective theory has electric charge 2e. In condensed matter physics, liquid metallic deuterium hosts both a Bose-Einstein condensate (BEC) as well as Cooper pairs [4]. Such a system can be described by two coupled fields with opposite signs for their respective electric charges. Mixtures of condensates of different electric charges is possible also in ultra-cold atomic gases, with a synthetic gauge field, see e.g. ref. [5]. Two-dimensional Skyrmions induced by quadratic band touching fermions can provide superconductivity with charge $4 e$ instead of $2 e$ [6]. These facts motivate the study of Abelian-Higgs type models with generic electric charges. Condensed matter systems with two superconductors separated physically by a thin insulating or weakly superconducting layer are described by a two-component Abelian Higgs model, see refs. $[8,9]$. The case of a two-component superconductor with generic charges has been studied already by Garaud and Babaev [7].

This physical setup of two superconductors separated by a thin layer houses a seminal effect called the Josephson effect, where a superconducting current is passing through the thin layer and is proportional to sine of the so-called Josephson phase [10, 11]. The Josephson phase is defined as the difference between the arguments of the order parameters of the two respective superconductors sandwiching the thin insulating layer. The effect can be understood quantum mechanically as a tunneling of a Cooper pair across the thin layer and is described in the two-component Abelian-Higgs model by the so-called Josephson term. Because the effect is really quantum mechanical - although macroscopically observable - it is possible to construct a single-electron transistor using the Josephson junction [12]. Other interesting applications are superconducting quantum interference devices called SQUIDs, as well as qubits which are the integral part of current day's quantum computing, see ref. [13] for a review.

The Josephson term also arises in two-component Bose-Einstein condensates (BECs) as an intrinsic interaction between two different states of the same condensate, propagated by an external field, as opposed to the Josephson junction, described above, where the interaction comes from a tunneling effect between two different condensates (order parameters). The former is called a Rabi term for BECs [14-16] and an intrinsic Josephson term for type-II superconductors. Josephson terms have been also studied in field-theoretical setups [17-19], and have been generalized to non-Abelian Josephson junctions [20, 21] and intrinsic interactions [22]. ${ }^{1}$

Sine-Gordon solitons often exist in two-component models possessing Josephson terms and live in Josephson phase function [26, 27]. They have also been found in one-dimensional type-II superconductors without a Josephson junction [28] and in two-component BECs [29]. Vortices in each component are fractionally quantized in the sense that integer winding numbers of the complex fields at spatial infinity lead to a fractional magnetic flux $[4,30]$.

\footnotetext{
${ }^{1}$ The Josephson interaction term also appears naturally in two-Higgs doublet models (2HDMs) in particle physics (see ref. [23] for a review), which can affect vortices and give rise to domain walls [24, 25].
} 
The presence of Josephson terms induces attraction between the vortices of the two different components, which creates the possibility of fractional vortex molecules $[29,31]$. Fractional vortex molecules are subject to vortex confinement [32-34] and can give rise to vortex lattices [35] as well as Berezinskii-Kosterlitz-Thouless transitions [36].

In this paper, we consider the marriage of the two concepts: namely the possibility of having generic charges of the two order parameters in a two-flavor model (or two-component superconductor) and having a Josephson term. This has not been considered previously, for good reasons, because the Josephson term only allows the two order parameters to have the same charges. In this paper we propose a generalization of the Josephson term to arbitrary integer charges of the same sign, which is gauge invariant, but does not have the physical interpretation of a Cooper pair tunneling across a thin barrier, unless the two charges are both equal to 2 (from now on, we will only specify the integer coefficient of the charge $e$ ).

Our first result is that the total winding number $k$, is a rational fraction when the vortices are local - i.e. when $n_{\text {global }}=0$, but is arbitrarily fractional (could be irrational) when the vortices are global - i.e. when $n_{\text {global }} \neq 0$. The latter situation yields angular domain walls, which we find analytic approximations for. The angular domain walls live in the generalized Josephson phase, which we denote the global vortex phase.

Next, we find that the only finite-energy vortices that exist in our model are local vortices - i.e. with $n_{\text {global }}=0$. If the vortices are global, on the other hand, the energy is logarithmically divergent (in the plane) without the new generalized Josephson term and linearly divergent with it. The latter linear divergence is simply due to the presence of the angular domain walls emanating from the vortex cores of the global vortices.

Finally, we perform numerical calculations and find a full classification of the minimal local vortices in the theory for electric charges up to four. We also illustrate some nonminimal local vortices that come in two variants of infinitely extendable networks. Finally, we make a numerical comparison between the angular domain walls and the analytic solution.

The paper is organized as follows. Section 2 introduces the model, the symmetries in section 2.1 and the vacuum in section 2.2. The vortex Ansatz is used to construct local and global vortices in section 2.3 with and without the generalized Josephson term and in the former case, an approximation makes it possible to find an explicit analytic solution for the so-called angular domain wall, that exists for global vortices. Finite energy considerations are made in section 2.4. The results are presented in section 3, first describing the numerical method (section 3.1), the visualization scheme (section 3.2) and some intuition for constructing solutions (section 3.3). Then local vortices are constructed in section 3.4, whose minimal solutions are illustrated in section 3.5 and nonminimal in section 3.6. The dependence of the ratio of the VEVs is explored in section 3.7. Global vortices are considered in section 3.8, where a numerical solution is compared with the analytic approximate solution of section 2.3. Finally, the paper is concluded with a discussion in section 4 . 


\section{The model, vortex asymptotics and angular domain walls}

The static energy for our model containing two complex scalar fields (two components) $\phi_{1}$ and $\phi_{2}$ with charges $Q_{1}$ and $Q_{2}$, respectively, reads

$$
E=\int d^{2} x\left[\frac{1}{4 e^{2}} F_{i j}^{2}+\left|D_{i} \phi_{f}\right|^{2}+\sum_{f=1}^{2} \frac{\lambda_{f}^{2}}{2}\left(\left|\phi_{f}\right|^{2}-v_{f}^{2}\right)^{2}-\gamma\left(\phi_{1}^{Q_{2}} \bar{\phi}_{2}^{Q_{1}}+\bar{\phi}_{1}^{Q_{2}} \phi_{2}^{Q_{1}}\right)+\eta\right]
$$

and the covariant derivatives are given by

$$
D_{i} \phi_{f}=\partial_{i} \phi_{f}-i Q_{f} A_{i} \phi_{f}, \quad(f \text { not summed over })
$$

with the spatial index $i$ running over 1,2 and the flavor index $f=1,2$. The coupling constants of the model are $e>0$ for the $\mathrm{U}(1)$ gauge coupling constant, $Q_{f} \in \mathbb{Z}_{>0}$ are positive integer charges, $\lambda_{f}>0$ for the two symmetry breaking potentials, $v_{f}>0$ for the vacuum expectation values of the norm of the complex scalar fields, and finally $\gamma$ for the generalized Josephson term. $\eta \in \mathbb{R}$ is real constant that will be adjusted so as to set the vacuum energy to zero. One reason for considering only positive charges $Q_{f} \in \mathbb{Z}_{>0}$ is that the generalized Josephson term is then well defined also at the points in space where $\sum_{g=1}^{2} \sigma_{f g}^{1} \phi_{g}$ vanishes, with

$$
\sigma_{f g}^{1}=\left(\begin{array}{ll}
0 & 1 \\
1 & 0
\end{array}\right)_{f g}
$$

being the first Pauli matrix and $f, g$ are the matrix indices.

The equations of motion read

$$
\begin{aligned}
D_{i}^{2} \phi_{f}-\lambda_{f}^{2}\left(\left|\phi_{f}\right|^{2}-v_{f}^{2}\right) \phi_{f}+\gamma \sum_{g=1}^{2} \sigma_{f g}^{1} Q_{g} \phi_{g}^{Q_{f}} \bar{\phi}_{f}^{Q_{g}-1}=0, \quad(f \text { not summed over }) \\
\partial_{i} F_{i j}-i e^{2} \sum_{f=1}^{2} Q_{f}\left(\bar{\phi}_{f} D_{j} \phi_{f}-\phi_{f}{\overline{D_{j} \phi_{f}}}\right)=0 .
\end{aligned}
$$

The generalized Josephson term will lead to a potential runaway instability (i.e. unbounded energy functional) for $Q_{f}>4$ or for $Q_{f}=4$ and $\gamma>\lambda_{f}^{2}$. For this reason, we will not consider charges larger than $Q_{f}=4$ for any $f$.

We note that the sign of the coefficient of the generalized Josephson term, $\gamma$, is unphysical. This can be seen readily by performing a global transformation of either of the complex scalar fields: e.g. take $\phi_{1}$ and make the transformation $\phi_{1} \rightarrow e^{i \frac{\pi}{Q_{2}}} \phi_{1}$, which changes $\gamma \rightarrow-\gamma$.

\section{$2.1 \quad$ Symmetries}

The symmetry of the model for $\gamma=0$ is

$$
G=\mathrm{U}(1)_{1} \times \mathrm{U}(1)_{2},
$$


which is a global symmetry

$$
\mathrm{U}(1)_{f}: \quad \phi_{f} \rightarrow e^{i \beta_{f}} \phi_{f}, \quad(f \text { not summed over })
$$

with $f=1,2$. Turning on $\gamma>0$ breaks the symmetry down to a subgroup - a combination of the two U(1)'s:

$$
\phi_{1} \rightarrow e^{i Q_{1} \beta} \phi_{1}, \quad \phi_{2} \rightarrow e^{i Q_{2} \beta} \phi_{2} .
$$

This is the combination that is gauged and thus the gauge transformation reads

$$
\phi_{1} \rightarrow e^{i Q_{1} \alpha(x)} \phi_{1}, \quad \phi_{2} \rightarrow e^{i Q_{2} \alpha(x)} \phi_{2}, \quad A_{i} \rightarrow A_{i}+\partial_{i} \alpha(x) .
$$

The model is invariant under this $\mathrm{U}(1)$ gauge transformation; in particular the generalized Josephson term is constructed on the principle of gauge invariance.

The breaking of the symmetry $G \rightarrow \tilde{G}$ is explicit (for $\gamma>0$ ) with

$$
\tilde{G}=\mathrm{U}(1)
$$

and this symmetry is then further completely broken spontaneously

$$
\tilde{G} \rightarrow H, \quad \text { with } \quad H=\mathbf{1} .
$$

Thus the topology of the vacuum supports topological vortices

$$
\pi_{1}(\tilde{G} / H)=\pi_{1}(\mathrm{U}(1))=\pi_{1}\left(S^{1}\right)=\mathbb{Z}
$$

Although the symmetry $G$ is explicitly broken, it will be useful to consider the generalized Josephson term as a perturbation and hence remember the original symmetry of the model, $G$, which gives rise to two distinct vorticities or vortex numbers

$$
\pi_{1}(G / H)=\pi_{1}\left(\mathrm{U}(1)_{1} \times \mathrm{U}(1)_{2}\right)=\mathbb{Z} \oplus \mathbb{Z}
$$

If we denote the two vortex numbers by $n_{f}$ :

$$
n_{f} \in \mathbb{Z}, \quad f=1,2,
$$

it will prove convenient to define two linear combinations

$$
\begin{aligned}
n_{\text {local }} & =Q_{2} n_{1}+Q_{1} n_{2}, \\
n_{\text {global }} & =Q_{2} n_{1}-Q_{1} n_{2} .
\end{aligned}
$$

The meaning of these new "vortex numbers" will become clear shortly; they can be viewed as the number of local and number of global vortices, respectively. 


\subsection{The vacuum}

Minimization of the Mexican hat potential (the third term in the energy (2.1)) leads to the vacuum solution

$$
\phi_{f}=v_{f} e^{i \theta_{f}} . \quad(f \text { not summed over })
$$

Substituting this into the generalized Josephson term (the fourth term in the energy (2.1)) gives

$$
-2 \gamma v_{1}^{Q_{2}} v_{2}^{Q_{1}} \cos \left(Q_{2} \theta_{1}-Q_{1} \theta_{2}\right) .
$$

The number of vacua is infinite, because we can shift both angles and get a "new vacuum": we will count such a vacuum as the same. Counting how many discrete possibilities the problem

$$
Q_{2} \theta_{1}-Q_{1} \theta_{2}=\frac{\pi}{2}+\mathrm{m} \pi
$$

with $\mathrm{m} \in \mathbb{Z}$ has for $\theta_{1,2} \in[0,2 \pi)$ yields the result

$$
\text { \# of vacua }=2 \max \left(Q_{1}, Q_{2}\right),
$$

where we recall that the charges are positive integers: $Q_{f} \in \mathbb{Z}_{>0}$. This means that the number of different domain walls is

$$
2 \max \left(Q_{1}, Q_{2}\right)-1
$$

The constant $\eta$ is thus determined as

$$
\eta=2 \gamma v_{1}^{Q_{2}} v_{2}^{Q_{1}} \cos \left(Q_{2} \theta_{1}-Q_{1} \theta_{2}\right),
$$

which vanishes $(\eta=0)$ for the $2 \max \left(Q_{1}, Q_{2}\right)$ solutions (2.19), but otherwise is generically equal to

$$
\eta=2 \gamma v_{1}^{Q_{2}} v_{2}^{Q_{1}} .
$$

\subsection{Vortex Ansatz}

\subsection{1 $\gamma=0$}

We consider the following axially symmetric Ansatz for vortices in both complex scalar fields and a corresponding transverse component of the gauge field.

$$
\begin{aligned}
\phi_{f} & =v_{f} h_{f}(r) e^{i n_{f} \theta}, \quad(f \text { not summed over }) \\
A_{i} & =-\frac{\varepsilon_{i j} x^{j}}{r^{2}} k a(r),
\end{aligned}
$$

with boundary conditions

$$
\begin{aligned}
h_{1,2}(0) & =0, & \lim _{r \rightarrow \infty} h_{1,2}(r) & =1, \\
a(0) & =0, & \lim _{r \rightarrow \infty} a(r) & =1 .
\end{aligned}
$$


Writing now the equations of motion (2.4)-(2.5) using the above Ansatz, we have

$$
\begin{gathered}
h_{f}^{\prime \prime}+\frac{1}{r} h_{f}^{\prime}-\frac{1}{r^{2}}\left(k a Q_{f}-n_{f}\right)^{2} h_{f}-\lambda_{f}^{2} v_{f}^{2}\left(h_{f}^{2}-1\right) h_{f} \\
+\gamma \sum_{g=1}^{2} \sigma_{f g}^{1} Q_{g} \cos \left[\left(Q_{2} n_{1}-Q_{1} n_{2}\right) \theta\right] v_{f}^{Q_{g}-2} v_{g}^{Q_{f}} h_{f}^{Q_{g}-1} h_{g}^{Q_{f}}=0, \quad(f \text { not summed over }) \\
\gamma \sum_{g=1}^{2} \epsilon_{f g} Q_{g} \sin \left[\left(Q_{2} n_{1}-Q_{1} n_{2}\right) \theta\right] v_{f}^{Q_{g}-2} v_{g}^{Q_{f}} h_{f}^{Q_{g}-2} h_{g}=0, \quad(f \text { not summed over }) \\
k^{2}\left(a^{\prime \prime}-\frac{1}{r} a^{\prime}\right)-2 e^{2} \sum_{f=1}^{2} v_{f}^{2} h_{f}^{2}\left(k a Q_{f}-n_{f}\right) k Q_{f}=0,
\end{gathered}
$$

where $h^{\prime} \equiv \partial_{r} h, h^{\prime \prime} \equiv \partial_{r}^{2} h$ and so on. Unless $Q_{2} n_{1}-Q_{1} n_{2}=n_{\text {global }}=0$, the generalized Josephson term is not compatible with the axially symmetric vortex Ansatz and we have to set $\gamma=0$ due to eq. (2.29), which reduces eq. (2.28) to:

$$
h_{f}^{\prime \prime}+\frac{1}{r} h_{f}^{\prime}-\frac{1}{r^{2}}\left(k a Q_{f}-n_{f}\right)^{2} h_{f}-\lambda_{f}^{2} v_{f}^{2}\left(h_{f}^{2}-1\right) h_{f}=0 . \quad(f \text { not summed over })
$$

Applying the boundary conditions (2.27) to eq. (2.30) yields an algebraic equation that can be solved for the total winding number, $k$, giving

$$
k=\frac{n_{1} Q_{1} v_{1}^{2}+n_{2} Q_{2} v_{2}^{2}}{Q_{1}^{2} v_{1}^{2}+Q_{2}^{2} v_{2}^{2}} .
$$

The total magnetic flux is thus

$$
\Phi=\int d^{2} x F_{12}=2 \pi k
$$

We will call a vortex configuration with this magnetic flux a fractional vortex, because $k$ is generically not an integer and not even necessarily a rational number. Rewriting the total winding number in terms of the local and global vortex numbers (2.15)-(2.16), we get

$$
k=\frac{1}{2 Q_{1} Q_{2}} n_{\text {local }}+\frac{Q_{1}^{2} v_{1}^{2}-Q_{2}^{2} v_{2}^{2}}{2 Q_{1} Q_{2}\left(Q_{1}^{2} v_{1}^{2}+Q_{2}^{2} v_{2}^{2}\right)} n_{\text {global }},
$$

from which it is clear that $n_{\text {global }}$ creates an arbitrary fraction, whilst $n_{\text {local }}$ yields a rational number.

Considering now the asymptotic behavior of the complex scalar fields, we apply the boundary conditions (2.27) to eq. (2.31) and obtain

$$
-\frac{1}{r^{2}} \sum_{g=1}^{2} \sigma_{f g}^{1}\left(\frac{n_{\text {global }} Q_{g} v_{g}^{2}}{Q_{1}^{2} v_{1}^{2}+Q_{2}^{2} v_{2}^{2}}\right)^{2} .
$$

This term comes from the mismatch of the gauge field asymptotically trying to cancel the contribution from the winding of the complex scalar fields. In fact, the presence of this term (i.e. for $n_{\text {global }} \neq 0$ ) will lead to a logarithmic divergence of the energy, see below. 


\subsection{2 $\gamma \neq 0$ : angular domain walls}

We will now consider the following axially symmetric vortex Ansatz, but with a nontrivial phase function and $\theta$ dependence:

$$
\begin{aligned}
\phi_{f} & =v_{f} h_{f}(r) e^{i n_{f} \Theta_{f}(r, \theta)}, \quad(f \text { not summed over }) \\
A_{i} & =-\frac{\varepsilon_{i j} x^{j}}{r^{2}} k a(r, \theta),
\end{aligned}
$$

where the boundary conditions now in addition to eq. (2.26) read

$$
\begin{aligned}
a(0, \theta) & =0, & \lim _{r \rightarrow \infty} a(r, \theta) & =1, \\
\Theta_{1,2}(r, 0) & =0, & \Theta_{1,2}(r, 2 \pi) & =2 \pi .
\end{aligned}
$$

Writing out the equations of motion now for the Ansatz (2.36)-(2.37) yields

$$
\begin{array}{r}
h_{f}^{\prime \prime}+\frac{1}{r} h_{f}^{\prime}-\frac{1}{r^{2}}\left(k a Q_{f}-n_{f} \dot{\Theta}_{F}\right)^{2} h_{f}-n_{f}^{2} h_{f}\left(\Theta_{f}^{\prime}\right)^{2}-\lambda_{f}^{2} v_{f}^{2}\left(h_{f}^{2}-1\right) h_{f} \\
+\gamma \sum_{g=1}^{2} \sigma_{f g}^{1} Q_{g} \cos \left(Q_{2} n_{1} \Theta_{1}-Q_{1} n_{2} \Theta_{2}\right) v_{f}^{Q_{g}-2} v_{g}^{Q_{f}} h_{f}^{Q_{g}-1} h_{g}^{Q_{f}}=0, \\
(f \text { not summed over }) \\
n_{f} \Theta_{f}^{\prime \prime}+\frac{1}{r} n_{f} \Theta_{f}^{\prime}+2 n_{f}\left(\log h_{f}\right)^{\prime} \Theta_{f}^{\prime}-\frac{1}{r^{2}} k Q_{f} \dot{a}+\frac{1}{r^{2}} n_{f} \ddot{\Theta}_{f} \\
-\gamma \sum_{g=1}^{2} \epsilon_{f g} Q_{g} v_{f}^{Q_{g}-2} v_{g}^{Q_{f}} h_{f}^{Q_{g}-2} h_{g} \sin \left(Q_{2} n_{1} \Theta_{1}-Q_{1} n_{2} \Theta_{2}\right)=0, \\
(f \text { not summed over }) \\
k\left(a^{\prime \prime}-\frac{a^{\prime}}{r}\right)-2 e^{2} \sum_{f=1}^{2} Q_{f} v_{f}^{2} h_{f}^{2}\left(k a Q_{f}-n_{f} \dot{\Theta}_{f}\right)=0,
\end{array}
$$

where $\epsilon_{f g}$ is the two-dimensional antisymmetric tensor with $\epsilon_{12}=1, \Theta^{\prime} \equiv \partial_{r} \Theta, \dot{\Theta} \equiv$ $\partial_{\theta} \Theta$ and so on. Since we are interested in the asymptotic behavior, we will consider the approximation of ignoring the radial derivatives. Suppressing the radial derivatives in eq. (2.42) and assuming that $h_{f}=1$, we get

$$
k a(\theta)=\frac{Q_{1} v_{1}^{2} n_{1} \dot{\Theta}_{1}(\theta)+Q_{2} v_{2}^{2} n_{2} \dot{\Theta}_{2}(\theta)}{Q_{1}^{2} v_{1}^{2}+Q_{2}^{2} v_{2}^{2}},
$$

which reduces to eq. (2.32) if

$$
\frac{1}{2 \pi} \int_{0}^{2 \pi} d \theta a=1
$$

Inserting eq. (2.43) into eq. (2.41), suppressing the radial derivatives and assuming $h_{f}=1$, yields

$$
\frac{Q_{2} n_{1} \ddot{\Theta}_{1}-Q_{1} n_{2} \ddot{\Theta}_{2}}{r^{2}\left(Q_{1}^{2} v_{1}^{2}+Q_{2}^{2} v_{2}^{2}\right)}-\gamma v_{1}^{Q_{2}-2} v_{2}^{Q_{1}-2} \sin \left(Q_{2} n_{1} \Theta_{1}-Q_{1} n_{2} \Theta_{2}\right)=0 .
$$


It will prove convenient to switch variables to

$$
\begin{aligned}
n_{\text {local }} \Theta_{\text {local }} & =Q_{2} n_{1} \Theta_{1}+Q_{1} n_{2} \Theta_{2}, \\
n_{\text {global }} \Theta_{\text {global }} & =Q_{2} n_{1} \Theta_{1}-Q_{1} n_{2} \Theta_{2},
\end{aligned}
$$

for which eq. (2.45) becomes

$$
n_{\text {global }} \ddot{\Theta}_{\text {global }}-r^{2} \gamma\left(Q_{1}^{2} v_{1}^{2}+Q_{2}^{2} v_{2}^{2}\right) v_{1}^{Q_{2}-2} v_{2}^{Q_{1}-2} \sin \left(n_{\text {global }} \Theta_{\text {global }}\right)=0 .
$$

For convenience, we will define the variable

$$
\zeta \equiv \theta r \kappa \equiv \theta r \sqrt{\gamma\left(Q_{1}^{2} v_{1}^{2}+Q_{2}^{2} v_{2}^{2}\right) v_{1}^{Q_{2}-2} v_{2}^{Q_{1}-2}}
$$

in terms of which eq. (2.48) reads

$$
n_{\text {global }} \frac{d^{2} \Theta_{\text {global }}}{d \zeta^{2}}-\sin \left(n_{\text {global }} \Theta_{\text {global }}\right)=0 .
$$

This equation is the well-known sine-Gordon equation and the solutions to this equation are sine-Gordon domain walls - which we shall call angular domain walls ${ }^{2}$

$$
\begin{aligned}
\Theta_{\text {global }} & =\sum_{\mathrm{m}=0}^{n_{\text {global }}-1} \Theta_{H}\left(\theta-\frac{2 \pi \mathrm{m}}{n_{\text {global }}}\right) \Theta_{H}\left(\frac{2 \pi(\mathrm{m}+1)}{n_{\text {global }}}-\theta\right) \Theta_{\text {global }}^{\mathrm{m}}, \\
\Theta_{\text {global }}^{\mathrm{m}} & =\frac{4}{n_{\text {global }}} \arctan \left(\exp \left[\left(\theta-\vartheta_{\mathrm{m}}\right) r \kappa\right]\right)+\frac{2 \pi \mathrm{m}}{n_{\text {global }}}, \\
\vartheta_{\mathrm{m}} & \in\left(\frac{2 \pi \mathrm{m}}{n_{\text {global }}}, \frac{2 \pi(\mathrm{m}+1)}{n_{\text {global }}}\right), \quad \mathrm{m}=0,1,2, \ldots, n_{\text {global }}-1,
\end{aligned}
$$

where $\Theta_{H}$ is the Heaviside step function

$$
\Theta_{H}(x)=\int_{-\infty}^{x} d s \delta(s)
$$

$\vartheta_{m}$ are directional moduli and $\kappa$ is given by eq. (2.49).

A comment is in store about the approximation we have made by picking up only 3 terms of the imaginary part of the scalar field equation of motion (2.41). More precisely, we have used the two terms involving $\theta$ derivatives as well as the potential bit, but discarded the radial derivatives. The rationale for this, is that we expect the angular domain walls to be translational invariant in say $x$, which means that the angular function will only depend on $r^{2}$ via the prefactor of the double $\theta$ derivative. We check this statement a posteriori, and indeed the radial derivative of the solution (2.51) is exponentially suppressed at large $r$ and can thus safely be ignored in the asymptotic determination of the domain wall profile. It does, however, play a role for the domain wall shape near the vortex core and this region will also fix the apparent directional moduli of the angular domain walls. However, to leading order in the asymptotic expansion, we cannot determine such fine details.

\footnotetext{
${ }^{2}$ For $Q_{1}=Q_{2}=2$ which is the conventional superconductor, the angular domain wall is called a fluxon [26].
} 


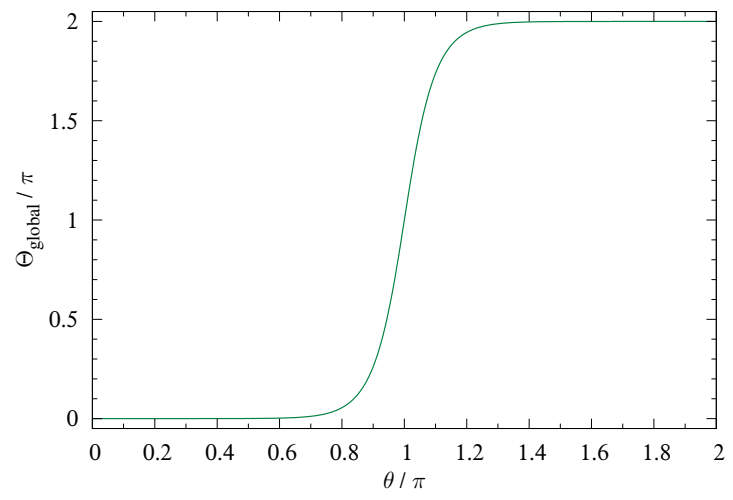

(a) $n_{\text {global }}=1$

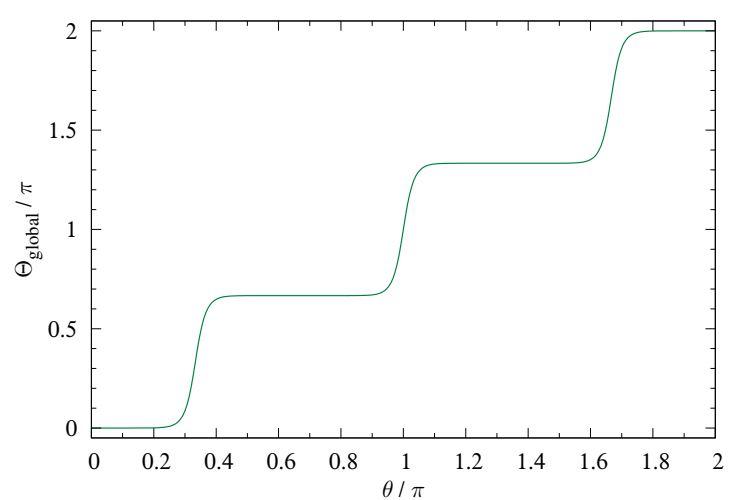

(c) $n_{\text {global }}=3$

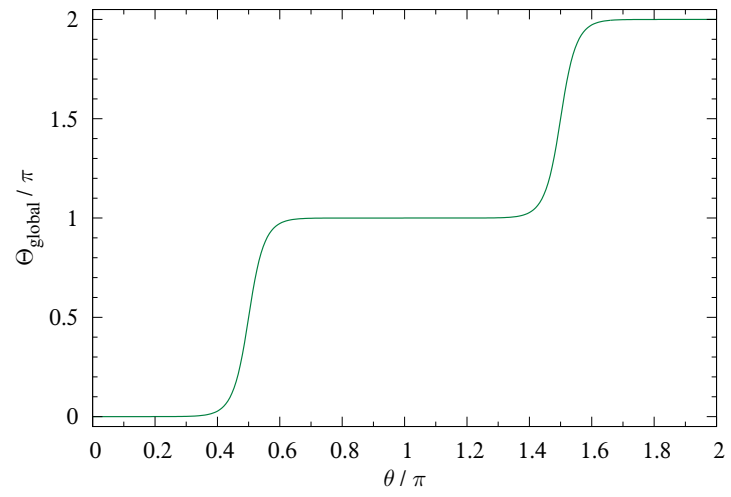

(b) $n_{\text {global }}=2$

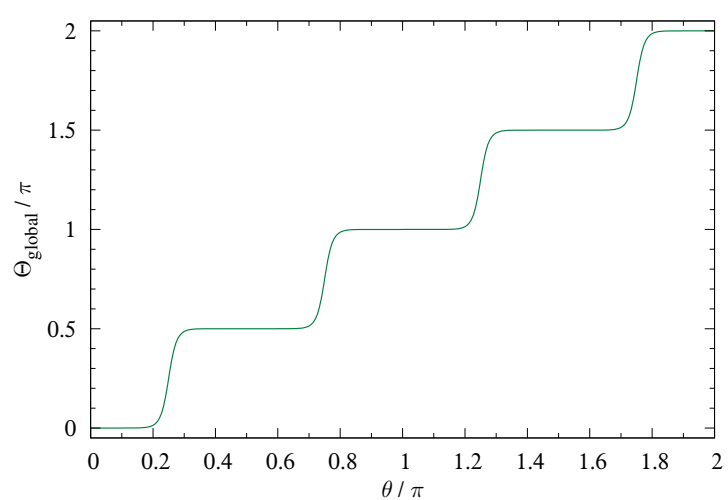

(d) $n_{\text {global }}=4$

Figure 1: Angular domain walls for $n_{\text {global }}=1,2,3,4$. For the figures, the following values where used: $r \kappa=5,10,15,20$ for the four panels, respectively, and the directional moduli were set to $\vartheta_{\mathrm{m}}=\pi(2 \mathrm{~m}+1) / n_{\text {global }}$, with $\mathrm{m}=0,1, \ldots, n_{\text {global }}$, which corresponds to the middle of their respective intervals (maximal repulsion).

Figure 1 shows examples of the angular domain wall solutions given in eq. (2.51). The solution is only valid asymptotically, i.e. for $r \kappa \gg n_{\text {global }}$.

Angular domain walls resemble axion domain walls emanating from an axion string in axion models (for a review, see e.g. ref. [37]), and axial domain walls from an axial vortex in QCD (for a review, see e.g. ref. [38]).

\subsection{Finite energy vortices}

We will now consider finiteness of the total energy in the plane. The exact behavior of the vortices near the origin and hence near their cores will not be important here, merely the behavior of the fields as they tend to spatial infinity.

\subsection{1 $\gamma=0$}

We start with the case of $\gamma=0$, for which the divergence was already spotted in eq. (2.35). For this analysis we again use the Ansatz (2.24)-(2.25) - which is appropriate for the $\gamma=0$ 
case - and insert it into the energy functional (2.1). Taking the asymptotic contributions to the energy integral one-by-one, yields

$$
\begin{aligned}
\frac{\pi}{2 e^{2}} \int^{R} d r r F_{i j}^{2} & \sim \text { finite, } \\
2 \pi \int^{R} d r r\left|D_{i} \phi_{f}\right|^{2} & \sim \frac{n_{\text {global }}^{2} v_{1}^{2} v_{2}^{2}}{\left(Q_{1}^{2} v_{1}^{2}+Q_{2}^{2} v_{2}^{2}\right)} 2 \pi \log R, \\
\pi \sum_{f=1}^{2} \lambda_{f}^{2} \int^{R} d r r\left(\left|\phi_{f}\right|^{2}-v_{f}^{2}\right)^{2} & \sim \text { finite, }
\end{aligned}
$$

with $R$ much bigger than any other scale in the system. It is clear that if we impose the condition that

$$
n_{\text {global }}=0, \quad \Rightarrow \quad n_{2} Q_{1}=n_{1} Q_{2},
$$

then the total energy is finite, even for infinitely large systems. Our first result of this paper is, however, this. Once we impose the condition, $n_{\text {global }}=0$ or equivalently $n_{2} Q_{1}=n_{1} Q_{2}$, the total winding number of the gauge field simplifies to

$$
k=\frac{1}{2 Q_{1} Q_{2}} n_{\text {local }}=\frac{n_{1}}{Q_{1}}=\frac{n_{2}}{Q_{2}},
$$

and is related to the magnetic flux by eq. (2.33). This winding number corresponds to a finite-energy configuration - we call a vortex with such a winding number a rational (fractional) vortex.

Notice that the finite-energy configurations do not necessarily have an integer times $2 \pi$ for the flux, but can take on any fractional value. Notice also that for any finite-energy configuration, the values of $v_{1,2}$ do not affect the total magnetic flux. For infinite-energy configurations - global vortices that have $n_{\text {global }} \neq 0$ - the ratio of $v_{1} / v_{2}$ can nevertheless affect the total magnetic flux unless $\left|Q_{1} v_{1}\right|=\left|Q_{2} v_{2}\right|$, for which an accidental cancellation makes gauge field unaware of the global vortex winding in the scalar fields.

\subsection{2 $\gamma \neq 0$ : angular domain walls}

Turning to the case of $\gamma \neq 0$, axial symmetry of the vortices is lost and the phase functions of the scalar fields, $\Theta_{1,2}$ - see eq. (2.36), develop nontrivial behavior described by angular domain walls (2.51). For this analysis, we will insert the Ansatz (2.36)-(2.37) into the energy functional (2.1). Taking again the asymptotic contributions to the energy one-byone, starting with the kinetic terms, yields

$$
\begin{aligned}
\frac{\pi}{2 e^{2}} \int^{R} d r r F_{i j}^{2} & \sim \text { finite } \\
\int^{R} d r r \int_{0}^{2 \pi} d \theta\left|D_{i} \phi_{f}\right|^{2} & \sim \int^{R} d r \int_{0}^{2 \pi} d \theta \frac{1}{r} \sum_{f=1}^{2} v_{f}^{2}\left(k a Q_{f}-n_{f} \dot{\Theta}_{f}\right)^{2} h_{f}^{2}+\text { finite } \\
& \sim \int^{R} d r \int_{0}^{2 \pi} d \theta \frac{v_{1}^{2} v_{2}^{2} n_{\text {global }}^{2} \dot{\Theta}_{\text {global }}^{2}}{r\left(Q_{1}^{2} v_{1}^{2}+Q_{2}^{2} v_{2}^{2}\right)}
\end{aligned}
$$




$$
\begin{aligned}
& \sim 4 \gamma v_{1}^{Q_{2}} v_{2}^{Q_{1}} \int^{R} d r \int_{0}^{\frac{2 \pi}{n_{\text {global }}}} d \theta r \sum_{\mathrm{m}=0}^{n_{\text {global }}-1} \operatorname{sech}^{2}\left[\left(\theta-\tilde{\vartheta}_{\mathrm{m}}\right) r \kappa\right] \\
& \sim \frac{4 \sqrt{\gamma} v_{1}^{\frac{Q_{2}}{2}+1} v_{2}^{\frac{Q_{1}}{2}+1}}{\sqrt{Q_{1}^{2} v_{1}^{2}+Q_{2}^{2} v_{2}^{2}}} \int^{R} d r \sum_{\mathrm{m}=0}^{n_{\text {global }}-1}\left[\tanh \left(\left(\theta-\tilde{\vartheta}_{\mathrm{m}}\right) r \kappa\right)\right]_{0}^{\frac{2 \pi}{n_{\text {global }}}} \\
& \sim \frac{8 \sqrt{\gamma} v_{1}^{\frac{Q_{2}}{2}+1} v_{2}^{\frac{Q_{1}}{2}+1} n_{\text {global }}}{\sqrt{Q_{1}^{2} v_{1}^{2}+Q_{2}^{2} v_{2}^{2}}} R,
\end{aligned}
$$

where we have defined the shifted directional moduli

$$
\tilde{\vartheta}_{\mathrm{m}} \equiv \vartheta_{\mathrm{m}}-\frac{2 \pi \mathrm{m}}{n_{\text {global }}} .
$$

Turning now to the potentials, we have

$$
\begin{gathered}
\pi \sum_{f=1}^{2} \lambda_{f}^{2} \int^{R} d r r\left(\left|\phi_{f}\right|^{2}-v_{f}^{2}\right)^{2} \sim \text { finite } \\
\int^{R} d r r \int_{0}^{2 \pi} d \theta\left[\eta-\gamma\left(\phi_{1}^{Q_{2}} \bar{\phi}_{2}^{Q_{1}}+\bar{\phi}_{1}^{Q_{2}} \phi_{2}^{Q_{1}}\right)\right] \\
\sim \int^{R} d r r \int_{0}^{2 \pi} d \theta\left[\eta-2 \gamma v_{1}^{Q_{2}} v_{2}^{Q_{1}} \cos \left(n_{\text {global }} \Theta_{\text {global }}\right)\right] \\
\sim 4 \gamma v_{1}^{Q_{2}} v_{2}^{Q_{1}} \int^{R} d r r \int_{0}^{\frac{2 \pi}{n_{\text {global }}}} d \theta r \sum_{\mathrm{m}=0}^{n_{\text {global }}-1} \operatorname{sech}^{2}\left[\left(\theta-\tilde{\vartheta}_{\mathrm{m}}\right) r \kappa\right] \\
\sim \frac{8 \sqrt{\gamma} v_{1}^{\frac{Q_{2}}{2}+1} v_{2}^{\frac{Q_{1}}{2}+1} n_{\text {global }}}{\sqrt{Q_{1}^{2} v_{1}^{2}+Q_{2}^{2} v_{2}^{2}}} R
\end{gathered}
$$

Summing up all contributions, we have a linearly divergent energy

$$
E=\frac{16 \sqrt{\gamma} v_{1}^{\frac{Q_{2}}{2}+1} v_{2}^{\frac{Q_{1}}{2}+1} n_{\text {global }}}{\sqrt{Q_{1}^{2} v_{1}^{2}+Q_{2}^{2} v_{2}^{2}}} R+\mathcal{O}\left(\log R, R^{0}\right),
$$

if $\gamma \neq 0$ and $n_{\text {global }} \neq 0$.

Although it is clear that for $\gamma=0$ and $n_{\text {global }} \neq 0$, there is a logarithmic divergence, our analysis does not show whether there is a subleading logarithmic divergence in the case of $\gamma \neq 0$ and $n_{\text {global }} \neq 0$; that is, however, beyond the scope of this paper.

\section{Numerical results}

\subsection{Numerical method}

Our numerical calculations are carried out using a custom built CUDA C code for an NVIDIA GPU cluster and the code uses a simple gradient flow method to find the vortex solutions in the two-flavor Abelian-Higgs systems with a generalized Josephson interaction term. The static equations of motion are discretized using a fourth-order 5-point stencil of a standard finite difference scheme and the lattice points are updated using the fourth-order Runge-Kutta method. 


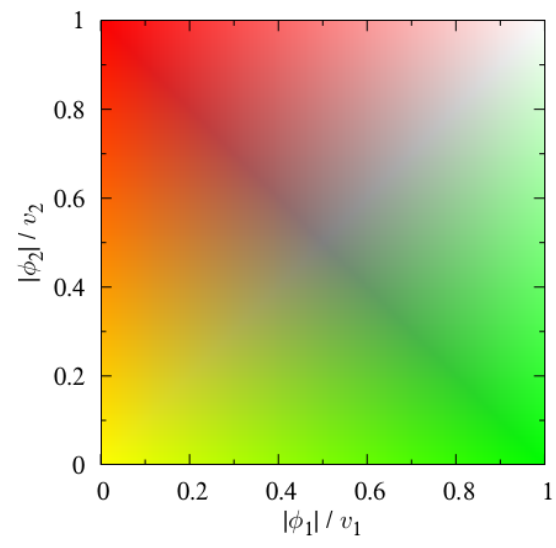

(a)

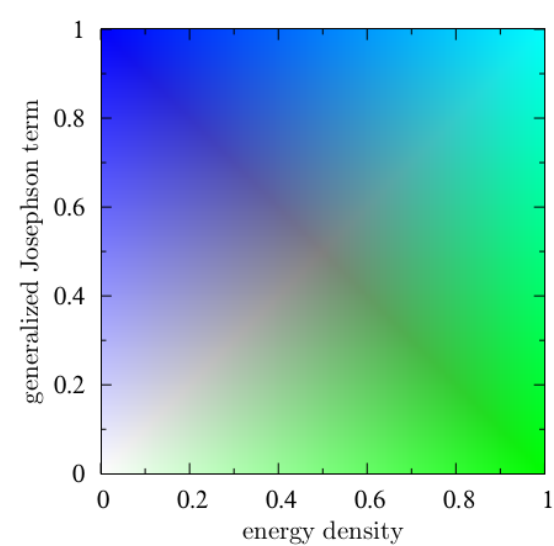

(b)

Figure 2: (a) Visualization of 2 complex fields using a customized coloring scheme. The vacuum is displayed with white, the vortices of $\phi_{1}$ with red, the vortices of $\phi_{2}$ with green and the coincident vortices of both $\phi_{1}$ and $\phi_{2}$ with yellow. (b) Visualization of the energy density and the generalized Josephson term (density) using a customized coloring scheme. The vacuum is displayed with white, the pure energy with green, the pure generalized Josephson term with blue and the overlap of the latter two with cyan.

\subsection{Visualization}

In order to visualize the results, we will employ a coloring scheme illustrated in figure 2 . The absolute values of the two complex scalar fields, $\left|\phi_{1,2}\right|$, are used via a 2 -dimensional coloring scheme illustrated in the figure to visualize the field configurations. The scheme is constructed such that the vacuum $\left(\left|\phi_{1}\right|=v_{1}\right.$ and $\left.\left|\phi_{2}\right|=v_{2}\right)$ is white, whereas the position of the vortices in $\phi_{1}$ (i.e. $\phi_{1} \approx 0$ ) are displayed with red and vortices in $\phi_{2}$ (i.e. $\phi_{2} \approx 0$ ) are displayed with green. The scheme is made such that the superposed vortices become yellow and the intermediate values of both fields turns out to become an interpolation function between red and green that goes through gray, see figure 2 .

\subsection{An intuitive explanation}

We will first and foremost be interested in local and hence finite-energy vortices, which poses the constraint $Q_{2} n_{1}=Q_{1} n_{2}$ (i.e. $n_{\text {global }}=0$ ) and in turn the winding number compensated by the gauge field is not just fractional, but rational, see eq. (2.58).

First we should mention that we will consider only the strong type II regime for both vortex species, which is tantamount to the conditions

$$
\lambda_{f} \gg e, \quad f=1,2 .
$$

This means that vortices of the same species will repel each other, but vortices with different species only interact with each other via the common gauge field and the generalized Josephson term $(\gamma \neq 0)$, see figure 3(a). 


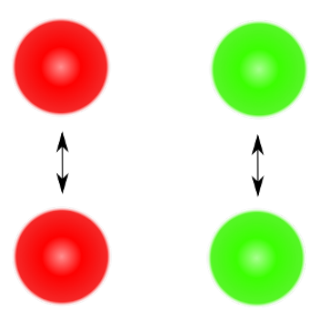

(a)

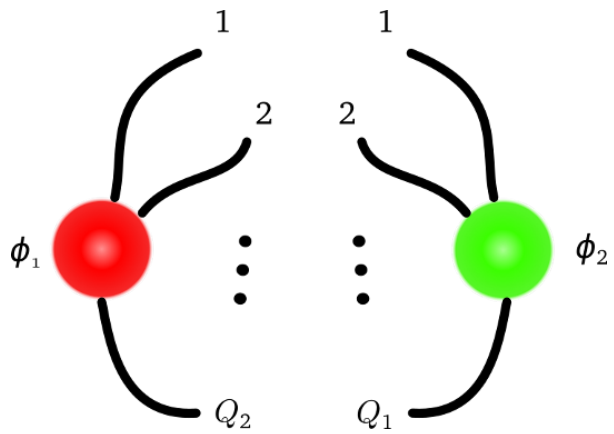

(b)

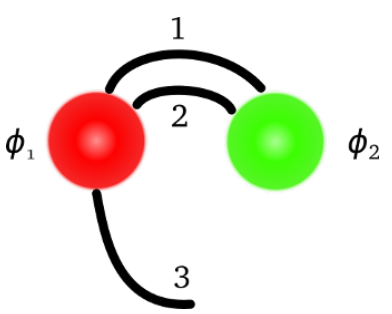

(c)

Figure 3: Intuitive explanation of building a vortex solution in our model. (a) Vortices of the same species repel each other, (b) but for $\gamma \neq 0, \phi_{1}$ has $Q_{2}$ legs while $\phi_{2}$ has $Q_{1}$ legs. (c) The connected legs attract the two vortices of opposite species in order to minimize the angular domain wall length. If there is a leg that cannot be connected anywhere, then $n_{\text {global }} \neq 0$ : in this example $Q_{2}=3, Q_{1}=2$ and $n_{1}=n_{2}=1$ so $n_{\text {global }}=Q_{2}-Q_{1}=1 \neq 0$.

In order to understand what the solutions of the model with $\gamma \neq 0$ look like, it will be illuminating to introduce a simple toy model, where each vortex in the field $\phi_{1}$ has $Q_{2}$ legs - corresponding to emanating angular domain walls - and each vortex of the field $\phi_{2}$ has $Q_{1}$ legs, see figure $3(\mathrm{~b})$.

In figure 3(c), we have made a simple example where the charges are assigned as $Q_{2}=3$ and $Q_{1}=2$, so that the red vortex species $(f=1)$ has three legs and the green vortex species $(f=2)$ has two legs. We can only connect legs from one vortex species to the opposite species. If any legs remain, the global vortex number is nonvanishing: the total energy will thus be linearly diverging, as demonstrated in section 2.4.2. In this simple example, there is one leg from the red vortex species remaining; thus the global vortex number, $n_{\text {global }}=1$. Had the remaining leg been emanating from the green vortex species, we should count it with a negative sign in the global vortex number and hence $n_{\text {global }}$ would be minus one instead.

Summary of how to construct vortices in our model:

1. Place $n_{1}$ red vortices and $n_{2}$ green vortices, each with $Q_{2}$ and $Q_{1}$ legs, respectively.

2. Connect as many legs between two opposite vortex species as possible.

(a) If no legs remain, we have successfully constructed a local vortex.

(b) If legs are remaining, the global vortex number is given by the number of legs from the red vortex species $(f=1)$ or minus the number of legs from the green vortex species $(f=2)$. 


\subsection{Constructing local vortices}

We will almost exclusively consider local vortex solutions in this paper, which entails solutions that obey

$$
n_{\text {global }}=Q_{2} n_{1}-Q_{1} n_{2}=0
$$

for which the "local" vortex number must be

$$
n_{\text {local }}=2 Q_{2} n_{1}=2 Q_{1} n_{2}
$$

and in turn the winding number compensated by the gauge field is given by eq. (2.58). The local vortex number is just a mathematically convenient construct, whereas physically the winding number $k$ is much more intuitive.

The minimally winding local vortices — which have finite energy and are spatially localized, have vortex numbers

$$
n_{1, \text { min }}=\frac{Q_{1}}{\operatorname{gcd}\left(Q_{1}, Q_{2}\right)}, \quad n_{2, \min }=\frac{Q_{2}}{\operatorname{gcd}\left(Q_{1}, Q_{2}\right)},
$$

for which the local vortex number is

$$
n_{\text {local }, \min }=\frac{2 Q_{1} Q_{2}}{\operatorname{gcd}\left(Q_{1}, Q_{2}\right)},
$$

while the winding number is

$$
k_{\min }=\frac{1}{\operatorname{gcd}\left(Q_{1}, Q_{2}\right)} .
$$

Obviously, we can have $n$ copies of such minimal local vortices, which just amounts to multiplying the above quantities by the integer $n \in \mathbb{Z}_{>0}$.

\subsection{Minimal solutions}

We will be interested only in the strong type-II domain where

$$
\frac{m_{\phi_{f}}}{m_{\gamma}}=\frac{\sqrt{2} \lambda_{f} v_{f}}{e \sqrt{Q_{1}^{2} v_{1}^{2}+Q_{2}^{2} v_{2}^{2}}} \gg 1, \quad(f \text { not summed over })
$$

for both $f=1,2$. For definiteness, we will choose

$$
\begin{aligned}
e & =0.3, \\
\lambda_{1}=\lambda_{2} & =2.5, \\
\gamma & =0.1,
\end{aligned}
$$

throughout the paper for all numerical calculations and also

$$
v_{1}=v_{2}=1,
$$




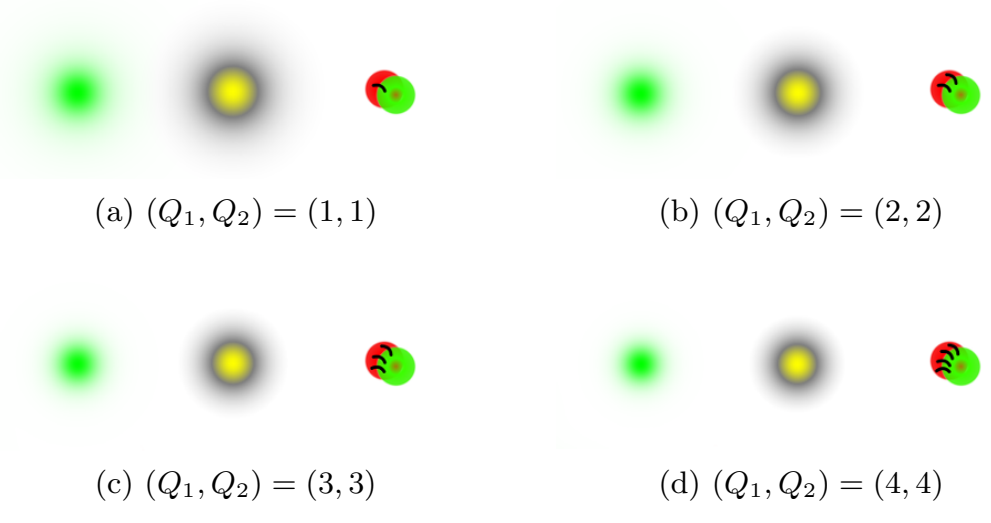

Figure 4: $Q_{1}=Q_{2}=Q$ minimal "normal" vortices with $Q=1,2,3,4$. Each subfigure contains 3 elements from left to right: an energy density plot, a field density plot and a sketch of how the red and green vortices are interconnected. The first two plots are made using the coloring scheme of figure 2 .

in this section, but we change their ratio in section 3.7. In order to retain stability of the vortex vacuum, we only consider the generalized Josephson term as a perturbation and hence take $\gamma \ll \lambda_{1,2}$, for which $\gamma=0.1$ is reasonable.

In this section, we will study the minimal solutions, by which we mean the minimal vortex numbers possible for a local vortex solution. This will in turn also result in the lowest energy solution - although for charges larger than 3, we begin to find energetically metastable solutions with the same vortex numbers as the global energy minimizing solution.

\subsection{1 $Q_{1}=Q_{2}=Q$ : the normal vortex}

The simplest case is when the two charges are equal. This means that the greatest common divisor is $\operatorname{gcd}\left(Q_{1}, Q_{2}\right)=Q$ and for $Q>1$ we have rational (fractional) vortices with $k=\frac{1}{Q}$. Nevertheless, since the two vortex species have the same number of legs, the minimal local vortex is simply given by connecting the $Q$ legs between two vortices of each species.

Figure 4 shows the minimal "normal" vortices for $Q=1,2,3,4$ and they are all just a pair of a vortex of each of the two species. Each subfigure shows the energy density and the field densities with the color schemes of figure 2 as well as a sketch of the vortex components and how they are connected with their legs.

The solutions thus look very similar and in fact the only difference between the solutions is the effective photon mass

$$
m_{\gamma}=e Q \sqrt{v_{1}^{2}+v_{2}^{2}}
$$

which changes with $Q$ and this in turn can be seen in figure 4 as different interpolations between yellow and gray (and then white being the vacuum).

The nontriviality of the local solutions will be seen first when the charges differ from unity and from each other; we will classify them in various categories next. 


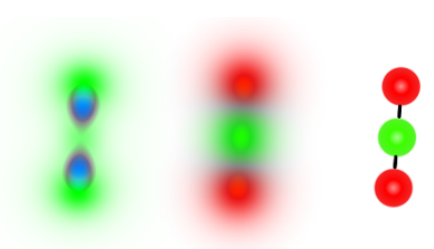

(a) $\left(Q_{1}, Q_{2}\right)=(2,1)$

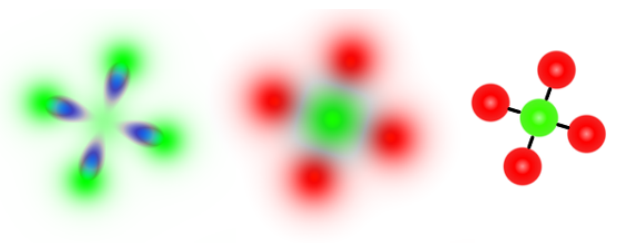

(c) $\left(Q_{1}, Q_{2}\right)=(4,1)$

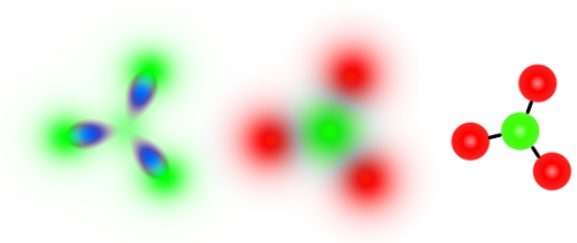

(b) $\left(Q_{1}, Q_{2}\right)=(3,1)$

Figure 5: $\left(Q_{1}, Q_{2}\right)=(Q, 1)$ minimal vortex "flowers", with $Q=2,3,4$. Each subfigure contains 3 elements from left to right: an energy density plot, a field density plot and a sketch of how the red and green vortices are interconnected. The first two plots are made using the coloring scheme of figure 2 .

\subsection{2 $\left(Q_{1}, Q_{2}\right)=(Q, 1)$ : the vortex flower}

The simplest nontrivial minimal vortex exists in the theory with one of the charges equal to unity and the other larger: thus we can take $\left(Q_{1}, Q_{2}\right)=(Q, 1)$ for $Q>1$. The vortex of the second species (green) will now have $Q$ legs and thus naturally be positioned in the center of the local vortex with $Q$ vortices of the first species (red) as petals around a receptacle.

Figure 5 displays the minimal "flower" vortices for $Q=2,3,4$. Each subfigure shows the energy density with the density of the generalized Josephson term overlaid using the color scheme of figure 2(b), the field densities using the color scheme of figure 2(a) as well as a sketch of the vortex components and how their legs are connecting them. As anticipated, the solutions look like flowers with $Q$ petals on a single receptacle. The legs are clearly visible on energy part of the subfigures as overlaid density of the generalized Josephson term.

One may speculate what if we were to increase $Q$ : would the solution still look like a flower? First of all, if $Q$ is larger than 4, stability is lost for any value of $\gamma$, as a runaway direction is opened up. The second issue is the bond length of the petal to the receptacle being model dependent; meaning that it depends on the values of $\lambda_{f}$ and $e$, etc. Some exploration revealed that what happens if there is energetically no room for an extra petal, the solution turns into a flower with $Q-1$ petals and a vortex with both species as the receptacle.

\subsection{3 $\left(Q_{1}, Q_{2}\right)=(Q+1, Q)$ : the vortex stick}

The next class of minimal vortices appear for $\left(Q_{1}, Q_{2}\right)=(Q+1, Q)$ with $Q \geq 1$ : we call this class of solutions vortex "sticks". The solution looks like a stick with $Q+1$ red vortices 


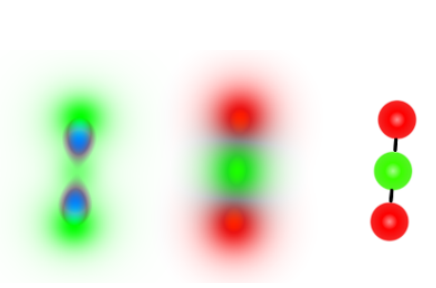

(a) $\left(Q_{1}, Q_{2}\right)=(2,1)$

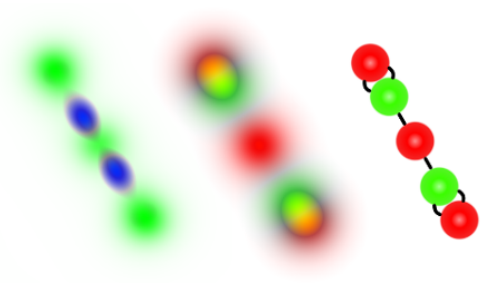

(b) $\left(Q_{1}, Q_{2}\right)=(3,2)$

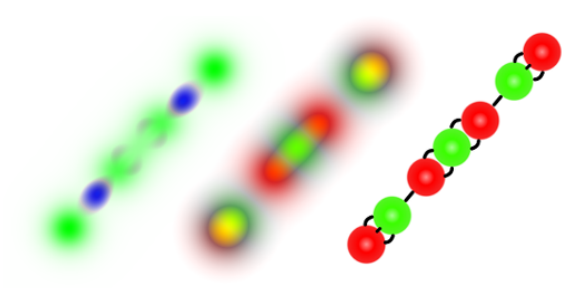

(c) $\left(Q_{1}, Q_{2}\right)=(4,3)$

Figure 6: $\left(Q_{1}, Q_{2}\right)=(Q+1, Q)$ vortex "sticks", with $Q=1,2,3$. Each subfigure contains 3 elements from left to right: an energy density plot, a field density plot and a sketch of how the red and green vortices are interconnected. The first two plots are made using the coloring scheme of figure 2 .

and $Q$ green vortices of alternating colors. Notice that for $Q=1$, the solution is exactly the same as the vortex "flower" with two petals.

Figure 6 shows the vortex "sticks" with $Q=1,2,3$. Each subfigure shows the energy densities with overlaid density of the generalized Josephson term, the field densities as well as a sketch of the leg connections. For $Q=3$, this is not the only possibility as we will see shortly.

For this class, we see for the first time the situation where more than one leg is connecting two adjacent vortices of opposite species. In figure 6(b), the double "bond" appears twice and is artistically drawn on the sketch on the sides of the two vortices; nevertheless, the overlay of the density of the generalized Josephson term clearly shows that the binding "bond" does indeed look like two separated legs. We should also notice that the length of the "bonds" for the double bond case is shorter than in the singlebond case. This trend continues to the triple-bond case where, unfortunately, it becomes impossible to see the density of the generalized Josephson term overlaying the total energy density. This is because the bond length is extremely short and the energy density is locally too small compared to that of the single bond.

\subsection{4 $\left(Q_{1}, Q_{2}\right)=(2 Q, Q)$ : the vortex pill}

As we cannot take the charges to be too large, we have not yet seen an example where the charges have a common factor. The simplest case and the only possible one for charges less-or-equal to four is the case of $\left(Q_{1}, Q_{2}\right)=(4,2)$, which thus can attain the solution of the $\left(Q_{1}, Q_{2}\right)=(2,1)$ model by doubling the number of legs between the vortices. We will 


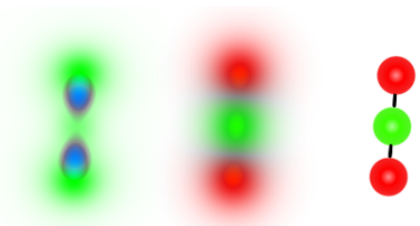

(a) $\left(Q_{1}, Q_{2}\right)=(2,1)$

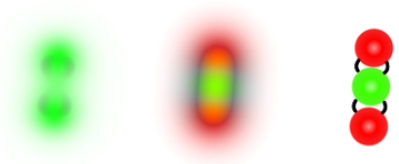

(b) $\left(Q_{1}, Q_{2}\right)=(4,2)$

Figure 7: $\left(Q_{1}, Q_{2}\right)=(2 Q, Q)$ minimal vortex "pills", with $Q=1,2$. Each subfigure contains 3 elements from left to right: an energy density plot, a field density plot and a sketch of how the red and green vortices are interconnected. The first two plots are made using the coloring scheme of figure 2 .

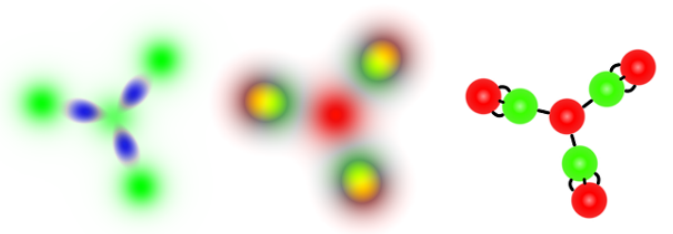

Figure 8: $\left(Q_{1}, Q_{2}\right)=(4,3)$ minimal vortex "extended flower", which is the minimumenergy local vortex in its charge sector. The figure contains 3 elements from left to right: an energy density plot, a field density plot and a sketch of how the red and green vortices are interconnected. The first two plots are made using the coloring scheme of figure 2.

call this type, the vortex "pill", which is nothing but the simplest vortex "flower" with only two petals or the simplest vortex "stick".

Figure 7 shows the minimal vortex "pills" for $Q=1,2$. Each subfigure shows the energy density with overlaid density of the generalized Josephson term, the field densities as well as a sketch of the leg connections. The double bonds in figure 7(b) versus the single bonds in figure $7(\mathrm{a})$ are clearly visible in the energy part of the subfigures.

\subsection{5 $\left(Q_{1}, Q_{2}\right)=(4,3)$ : the extended flower}

So far all the minimal vortices have been the local vortex solutions with the smallest possible vortex numbers for given electric charges. Additionally, they have also been the global energy minimizing solutions for all cases but one - namely the $\left(Q_{1}, Q_{2}\right)=(4,3)$ case: in this case, the vortex "stick" is not the energetically favorable solution, but a slightly lower total energy can be obtained by deforming the stick into a "flower" form.

Figure 8 shows the lowest-energy $\left(Q_{1}, Q_{2}\right)=(4,3)$ local vortex, which takes the form of a flower with composite petals on a single-vortex receptacle. Each subfigure shows the energy density with overlaid density of the generalized Josephson term, the field densities as well as a sketch of the leg connections. The composite petals are made of two vortices one of each species - and they are connected internally by a triple bond. Unfortunately, the short triple bond is not visible on the energy part of the figure, whereas the single bonds are very clearly marked in the overlaid density of the generalized Josephson term. 


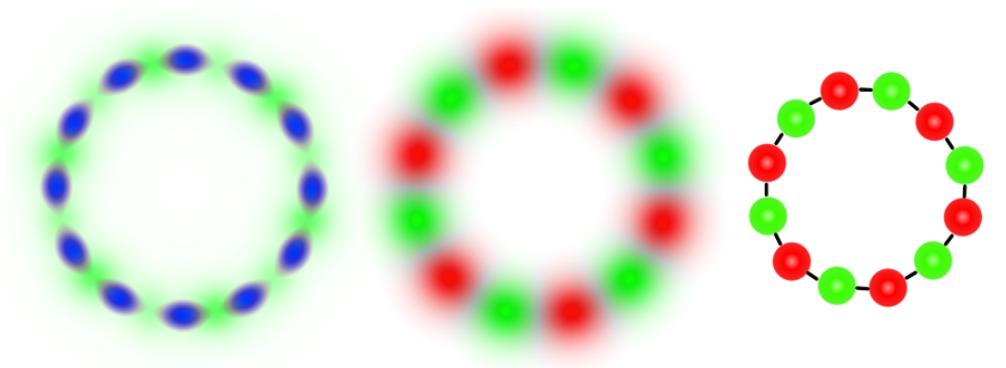

Figure 9: $\left(Q_{1}, Q_{2}\right)=(2,2)$ nonminimal vortex ring. The figure contains 3 elements from left to right: an energy density plot, a field density plot and a sketch of how the red and green vortices are interconnected. The first two plots are made using the coloring scheme of figure 2.

Although the composite petal is very compact, it is nevertheless possible to see on the field densities part of the figure that the green vortex is the inner-most one as it must connect to the red central receptacle.

\subsection{Nonminimal solutions}

It should be obvious by now that the minimal vortices cannot be the only possibilities of connecting the legs to form local vortices. We call the remaining infinity of solutions, nonminimal solutions. Indeed as there are infinitely many nonminimal solutions, we will only display a selected few solutions that we have found numerically.

Figure 9 shows the case with the smallest possible electric charges possessing nonminimal solutions, viz. the $\left(Q_{1}, Q_{2}\right)=(2,2)$ case. The minimal local vortex with these charges is simply a vortex of both species superposed one on the other, see figure 4(b). Instead of using two legs to connect the two vortices of opposite species, we can connect two green vortices to a single red vortex, which leaves one leg unconnected. The solution is simple: indeed we can make a ring network of vortices of alternating species (red, green, red, ..., green). The particular ring network solution found and illustrated in figure 9 contains six vortices of each species.

Although the red vortex and the green vortex (i.e. of species 1 and species 2, respectively) do not repel each other, the strong repulsive force between vortices of the same species push them as far apart as possible, turning the solution into a network of alternating vortex species on a wound-up string - a ring network. The reason for the whole construction not to fall apart, is that the legs contribute to the energy via the generalized Josephson term and hence must be shortened as much as possible. The balance act between the two opposing forces creates the bond length and in turn the specific solution depicted in the figure.

The next example of a nonminimal local vortex is for the $\left(Q_{1}, Q_{2}\right)=(3,2)$ case. The minimal local vortex with these charges is the vortex "stick", see figure $6(\mathrm{~b})$. The $Q=2$ stick (i.e. $\left.\left(Q_{1}, Q_{2}\right)=(Q+1, Q)=(3,2)\right)$ has two parts with double bonds as opposed to the $Q=1$ stick, which has only single bonds. The double bonds can thus be split and used 


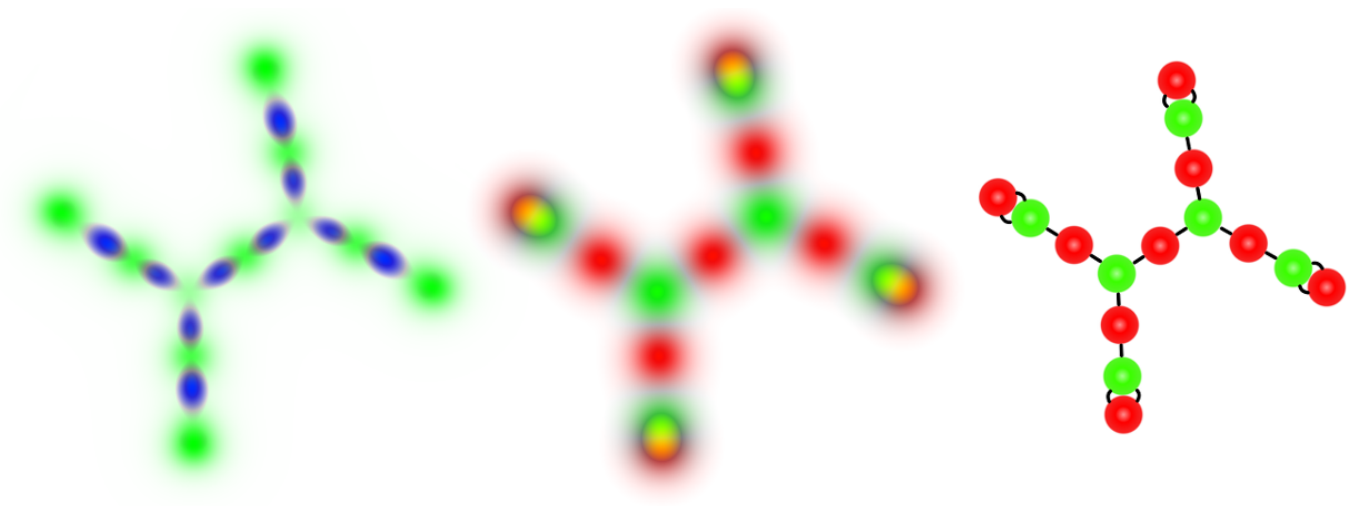

Figure 10: $\left(Q_{1}, Q_{2}\right)=(3,2)$ nonminimal vortex stick network. The figure contains 3 elements from left to right: an energy density plot, a field density plot and a sketch of how the red and green vortices are interconnected. The first two plots are made using the coloring scheme of figure 2 .

to connect other sticks and it is then unlimited how large a stick network one can build from this foundation.

The two basic building blocks in this construction are a vertex composed by a green vortex as a receptacle with three red petals, each having an unconnected leg, and the other is an end cap composed by a red and a green vortex interconnected by a double bond, leaving the green vortex with an unconnected leg. The two types of bond are clearly visible in figure 10 in the energy density part of the figure (the left-most part).

The specific vortex stick network depicted in figure 10 is composed by three minimal vortex sticks that have been deformed so as to form the stick network resembling the capital Latin letter 'H'.

The next example of a nonminimal local vortex is for the $\left(Q_{1}, Q_{2}\right)=(3,3)$ case. Since the two electric charges are equal, the minimal vortex is simply a vortex of each species, mutually interconnected by three legs, see figure 4(c). This triple bond can be split into a double bond with two loose legs or into a single bond with four loose legs. This complexity yields vortex ring network solutions with two basic components. The mentioned former option provides a link composed by a red and a green with a free leg from each one of them. The other basic component is the vertex, which is either a single red or a single green vortex with three legs connecting three chains of links.

Figure 11 shows the vortex ring network solution that we found numerically. Notice that at every vertex, the incoming legs must (of course) all be from the opposite vortex species, which in turn makes the chains of links directed graphs. It is definitely possible to make a simple ring out of a linked chain, which would be a simpler solution than the one depicted in figure 11. The above observation implies that once a vertex is inserted into a ring, an even number of vertices must be inserted in order to close the ring and hence make the vortex local. The double bonds in the links and the three single bond emanating from the vertices are clearly visible in blue on the energy density part of figure 11. 

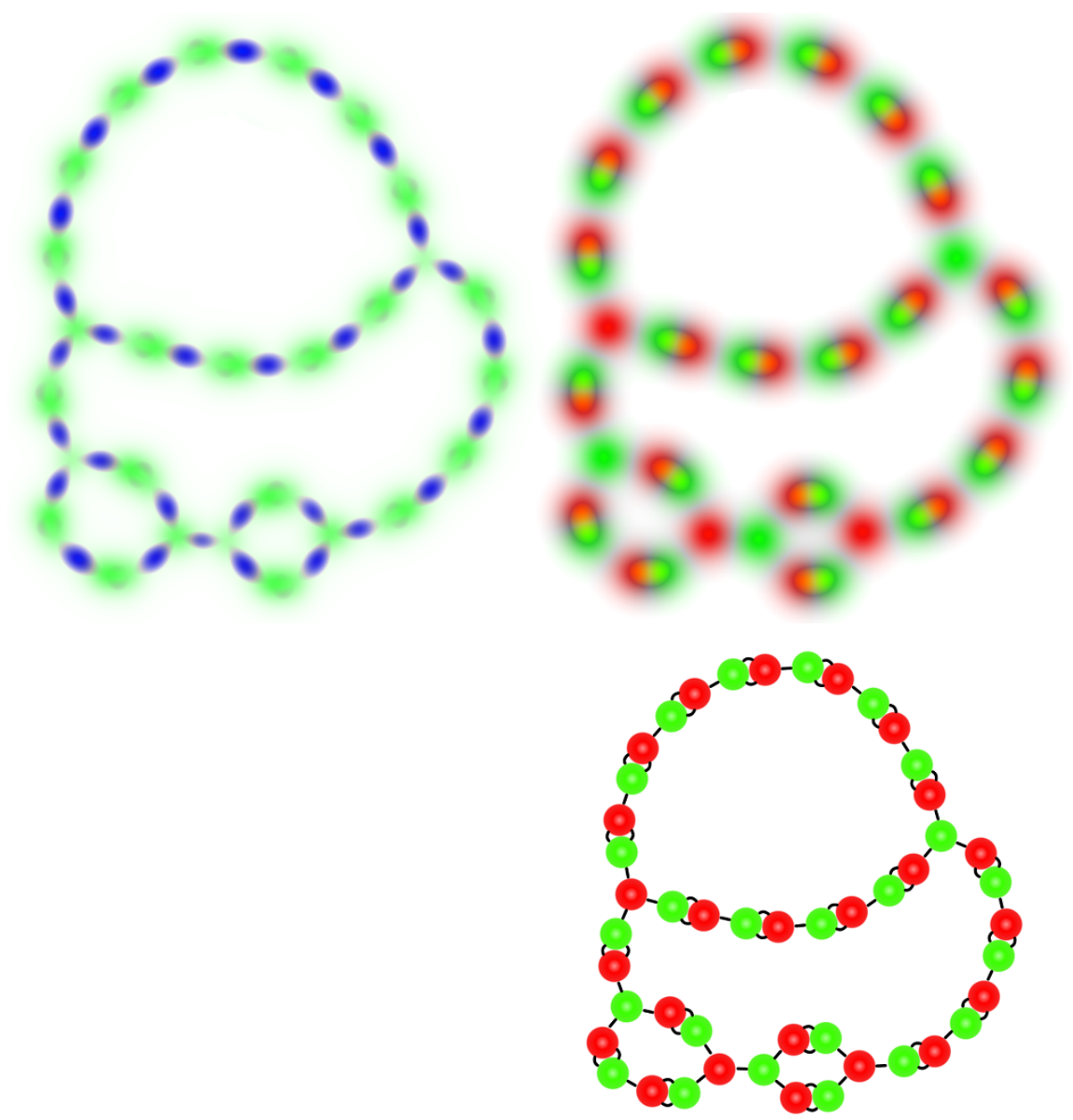

Figure 11: $\left(Q_{1}, Q_{2}\right)=(3,3)$ nonminimal vortex ring network. The figure contains 3 elements from left to right and then down: an energy density plot, a field density plot and a sketch of how the red and green vortices are interconnected. The first two plots are made using the coloring scheme of figure 2 .

The next example is the $\left(Q_{1}, Q_{2}\right)=(4,2)$ case, whose minimal local vortex solution is in the class of vortex "pills", see figure 7(b). The minimal local vortex has two double bonds and either double bond can be split into two single bonds making the green vortex a link piece with a red dweller on its back. There are thus two basic components in this model, the green link piece with a red dweller and simply a red vortex. Alternating these two components makes it possible to create a vortex ring network of any size.

Figure 12 shows a nonminimal vortex ring with seven links of both types described above. Since the dwelling red vortex is attached to the green, necessarily on one side of the green vortex (due to repulsion from the other red vortices in the ring network), the red dweller can either sit on the inside or the outside of the ring. The link naturally curves the ring away from the dweller and hence most dwellers are situated on the outside, since 

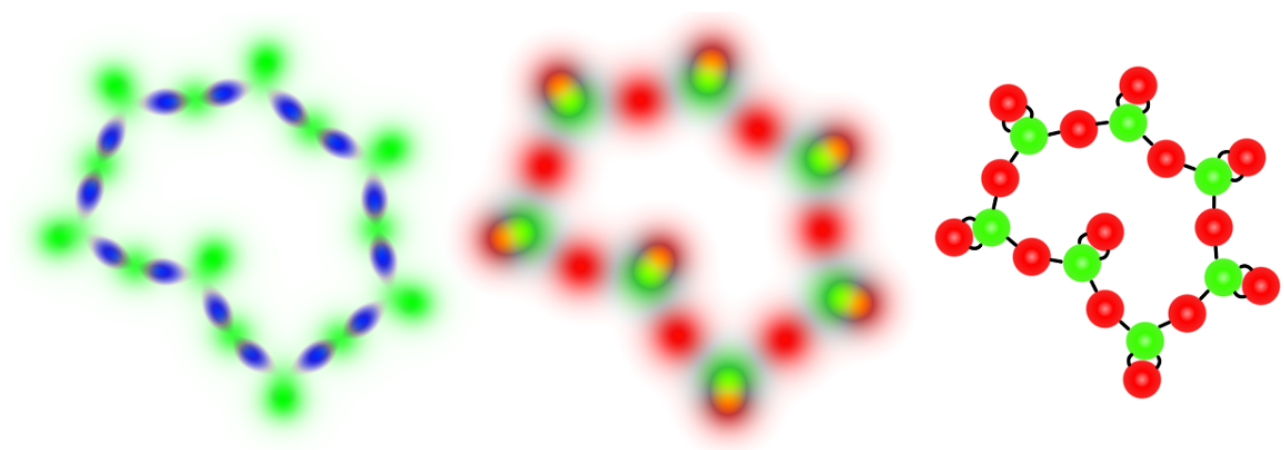

Figure 12: $\left(Q_{1}, Q_{2}\right)=(4,2)$ nonminimal vortex ring network. The figure contains 3 elements from left to right: an energy density plot, a field density plot and a sketch of how the red and green vortices are interconnected. The first two plots are made using the coloring scheme of figure 2 .

the overall curvature of the circle must be positive for the ring to close. Both the double bonds associated with the dweller and the single bond connecting the two types of link in the ring network of vortices are visible in the energy density part of figure 12 .

The next example is the $\left(Q_{1}, Q_{2}\right)=(4,3)$ case, whose minimal local vortex solutions are a "stick", see figure 6(c) and the "extended flower", see figure 8 - the latter of which has the lowest total energy. The $Q=3$ stick (i.e. $\left(Q_{1}, Q_{2}\right)=(4,3)$ ) has two triple bonds, two double bonds and two single bonds. Both the triple and double bonds can be split making the stick attachable to other sticks and thus provides the breeding ground for the vortex stick network.

Three basic building blocks can be made: an end cap which is a green and a red vortex interconnected by a triple bond, leaving the green vortex with a loose leg. The second building block is a link, which is made of two red vortices encapsulating a single green vortex with two double bonds, leaving both red vortices with a free leg each. The third and final building block is the vertex, which comes in two flavors: a three- and four-legged vertex; the formed is made by attaching a red vortex to the green vortex with a double bond, creating a vertex with two legs emanating from the green vortex and a single leg from the red vortex; the latter is simply the green vortex itself.

The numerically found vortex stick network in this model is shown in figure 13 and the single and double bonds are clearly visible on the energy density part (i.e. upper-left part) of the figure. Unfortunately, the end caps possessing triple bonds cannot be seen on the figure as the triple bonds are too short and weak in the energy density plots to be seen. The specific vortex stick network depicted in figure 13 is composed of four deformed minimal vortex sticks.

The final example of nonminimal local vortex solutions is in the $Q_{1}=Q_{2}=4$ model, whose minimal local vortex is simply a "normal" vortex composed of a red and green vortex interconnected by a quadruple bond (i.e. four legs), see figure 4(d). This quadruple bond can be split and thus the minimal vortex can be turned into components for building a vortex ring network. 

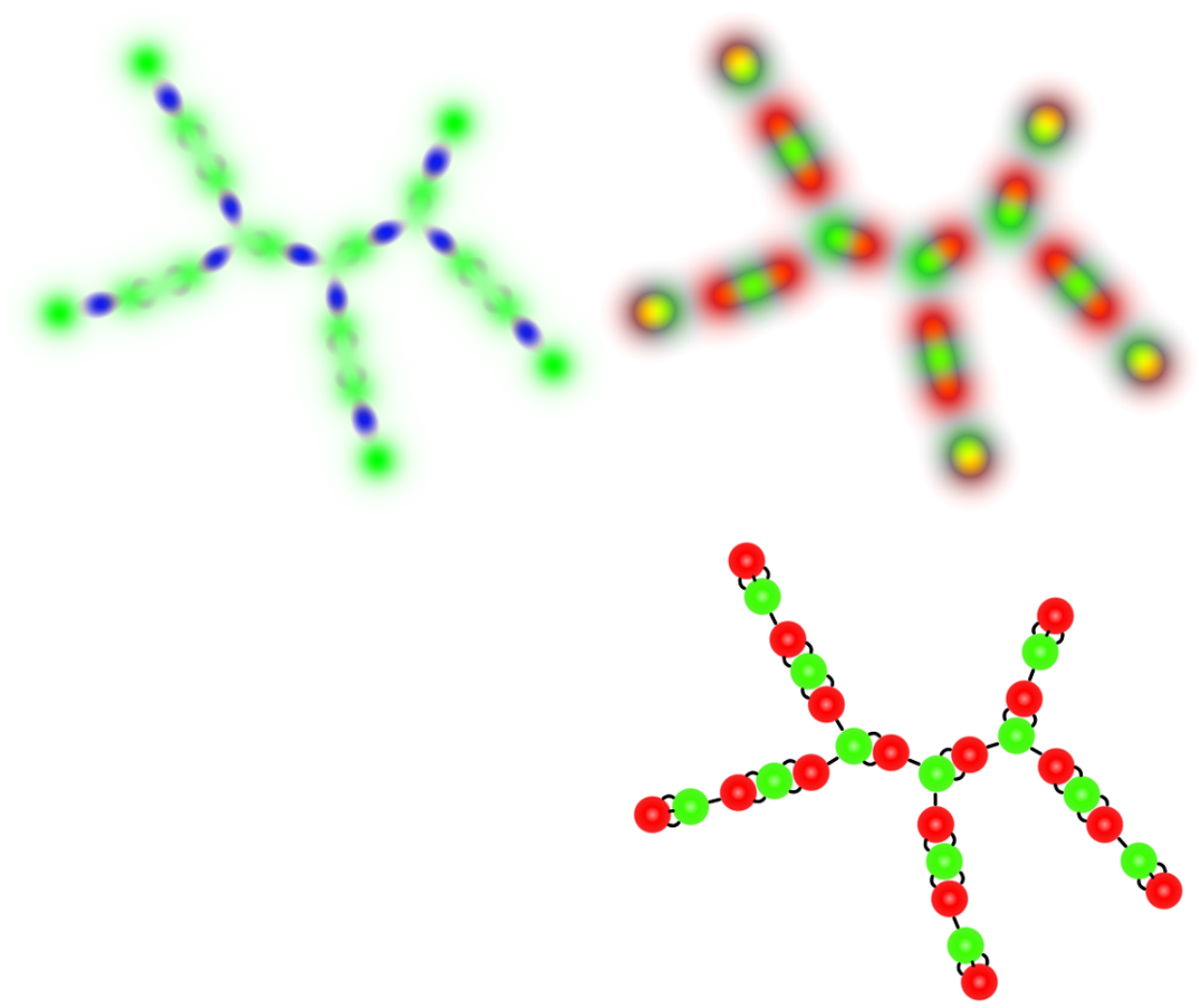

Figure 13: $\left(Q_{1}, Q_{2}\right)=(4,3)$ nonminimal vortex stick network. The figure contains 3 elements from left to right and then down: an energy density plot, a field density plot and a sketch of how the red and green vortices are interconnected. The first two plots are made using the coloring scheme of figure 2 .

The two basic building blocks are two types of links. The first type of link has a triple bond between a red and a green vortex, leaving a free leg on both the vortices. The other type of link possesses only a double bond, providing them with two free legs on either side. The second type of link can thus be connected to two links of the first type. In principle, vertices do also exist, but are not contained in the numerical solution displayed in figure 14 . Notice that by connecting two chains of the first type to on chain of the second type, the two chains of the first type necessarily have the same vortex species connecting the chain of the second type, and hence cannot be interconnected. Therefore, the chains of links are directed graphs and an even number of insertions of the second type of chain into the first type must occur. The numerically found solution of this model in figure 14 contains three chains of the first type and two chains of the second type, thus providing four insertions of chains of the second type into the ring of the first type. The single bonds linking the links of the first type and the double bonds in the chains of the second type are clearly visible in the energy density part of the figure. The triple bond possessed by the links of the first type are still not visible, but are illustrated in the sketch of the figure. 

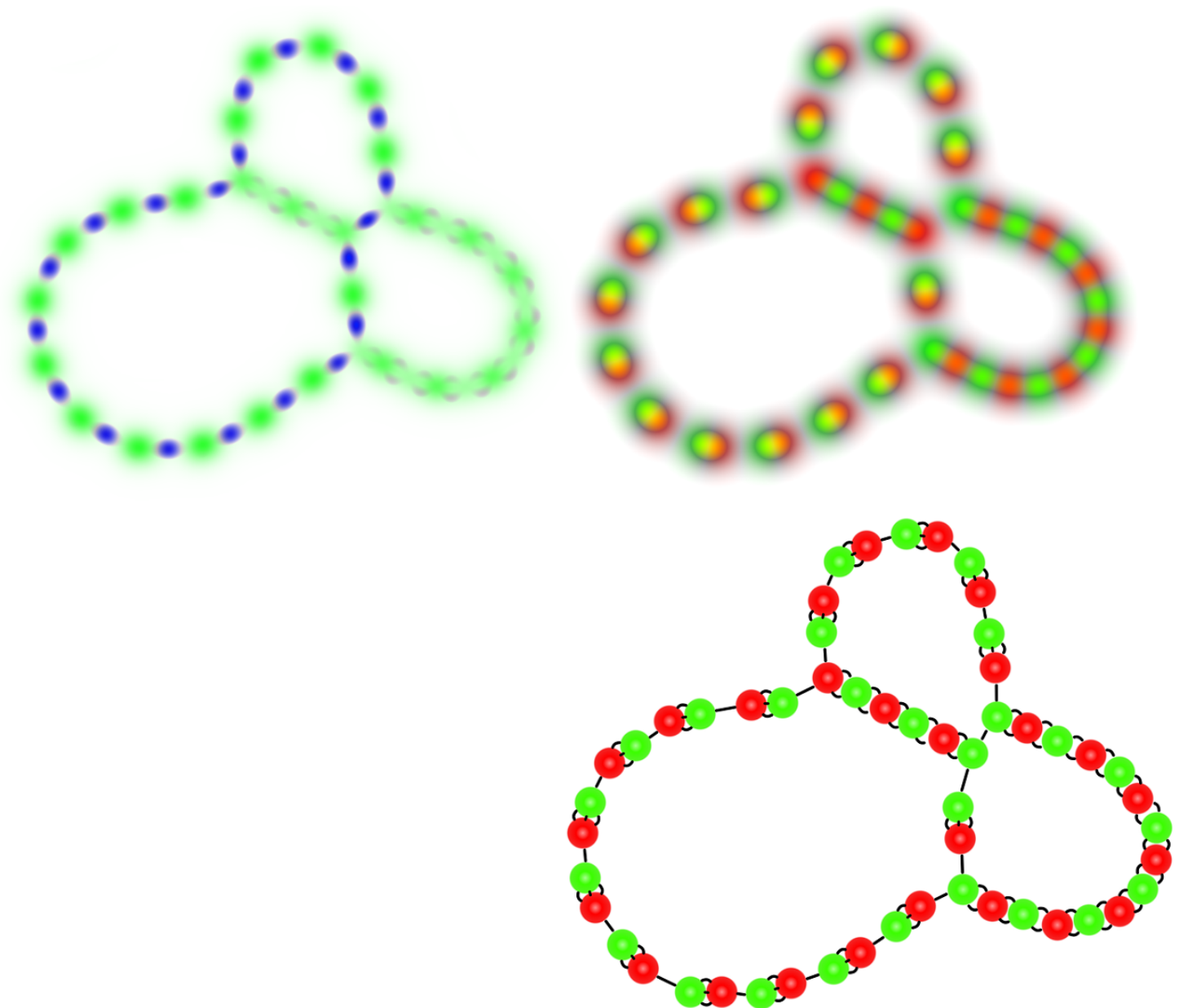

Figure 14: $\left(Q_{1}, Q_{2}\right)=(4,4)$ nonminimal vortex ring network. The figure contains 3 elements from left to right and then down: an energy density plot, a field density plot and a sketch of how the red and green vortices are interconnected. The first two plots are made using the coloring scheme of figure 2 .

\subsection{Varying the VEVs}

So far we have worked in a setting where $v_{1}=v_{2}=1$, which with $\lambda_{1}=\lambda_{2}$ provides a $\mathbb{Z}_{2}$ flavor symmetry between the vortices of the red and green species. In this section, we will illustrate how the vortex solutions change by varying the ratio of the VEVs, $v_{1} / v_{2}$. This could be done in various ways, but we choose to increase either of the two VEVs, $v_{1,2}$, because that would shrink the vortex of the given species (as opposed to lowering the VEV that would increase the vortex). This is due to the vortex length scales being

$$
\ell_{\phi_{f}}=m_{\phi_{f}}^{-1}=\frac{1}{\sqrt{2} \lambda_{f} v_{f}}, \quad(f \text { not summed over })
$$

for the scalar fields and

$$
\ell_{\gamma}=m_{\gamma}^{-1}=\frac{1}{e \sqrt{Q_{1}^{2} v_{1}^{2}+Q_{2}^{2} v_{2}^{2}}}
$$




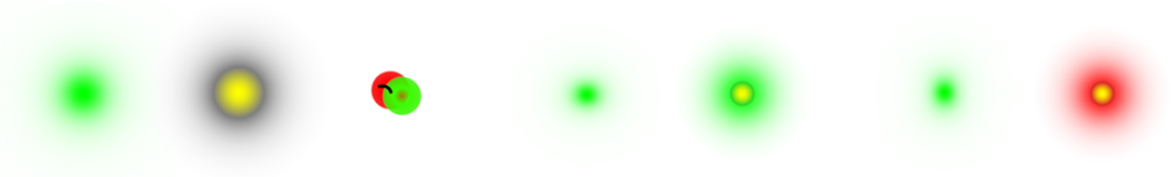

(a) $\left(Q_{1}, Q_{2}\right)=(1,1)$

Figure 15: $Q_{1}=Q_{2}=Q$ minimal "normal" vortex with $Q=1$. The figure contains 7 elements from left to right: for column 1 through $3: v_{1} / v_{2}=1$ : an energy density plot, a field density plot and a sketch of how the red and green vortices are interconnected. For column 4 and 5: $v_{1} / v_{2}=2$ : an energy density plot and a field density plot. For column 6 and 7: $v_{1} / v_{2}=1 / 2$ : an energy density plot and a field density plot. All columns except column 3 are made using the coloring scheme of figure 2 .

for the gauge field. This choice is simply because we use the solutions displayed in the previous section as seeds for the calculations and increasing the vortex sizes could make the solutions run out of space on the finite lattice used for the simulations.

We begin with the minimal "normal" vortex with electric charges $Q_{1}=Q_{2}=1$, whose solution is axially symmetric since it is made of a red and green vortex superposed at exactly the same position. We can confirm from figure 15, that the energy density (first column) is axially symmetric and the fields are coincident (second column), because the field plot is yellow turning into gray and then into white. The latter interpolation is exactly the diagonal of both fields vanishing and while being equal to each other, they increase to their common VEV. The third column of figure 15 shows the sketch of the red and the green vortex being interconnected by a single bond (leg). The fourth and fifth columns show the same vortex, but for $v_{1} / v_{2}=2$ making the red vortex smaller with respect to the equal VEV case shown in columns one and two. The result is that the yellow region of the field plot (fourth column) is small, but surrounded by a green cloud, as the green vortex is unaltered in size. We also notice that the energy density plot seems to have shrunk with respect to the equal VEV case; this is merely because the red vortex is smaller and hence its energy density is locally higher. It is still surrounded by a larger and weaker cloud of energy density coming from the green vortex. It can be somewhat hard to see, because the energy density plot is normalized so that the green color is the maximum local energy density. Finally, we increase the second VEV instead of the first, so that $v_{1} / v_{2}=1 / 2$, which is equivalent to swapping the red and the green vortex in the configuration. For this particular case, it is a triviality, and the result can be seen in columns six and seven of figure 15. In particular we can see that the small yellow region is now surrounded by a red cloud instead of a green cloud in the seventh column of the figure. For the remaining figures (except figure 9) in this section, $Q_{1} \neq Q_{2}$ and hence the sixth and seventh columns of the figure will be nontrivial compared with the fourth and fifth columns.

We now turn to varying the VEVs of the minimal vortex "flowers", see figure 16. As was the case of the previous figure and for the remaining figures of this section, the columns show the energy density with $v_{1} / v_{2}=1$, the field densities with $v_{1} / v_{2}=1$, the sketch of 

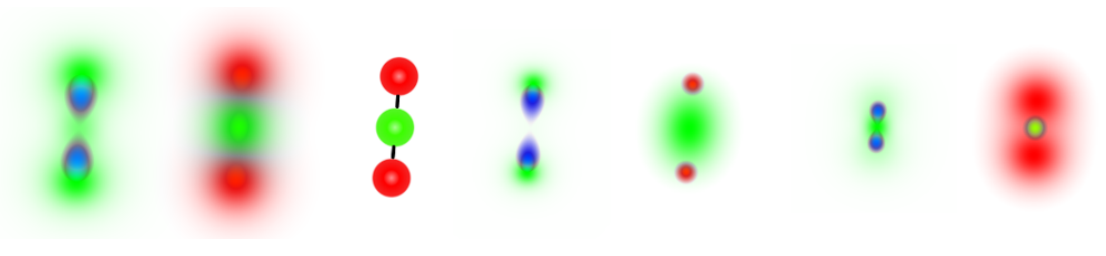

(a) $\left(Q_{1}, Q_{2}\right)=(2,1)$
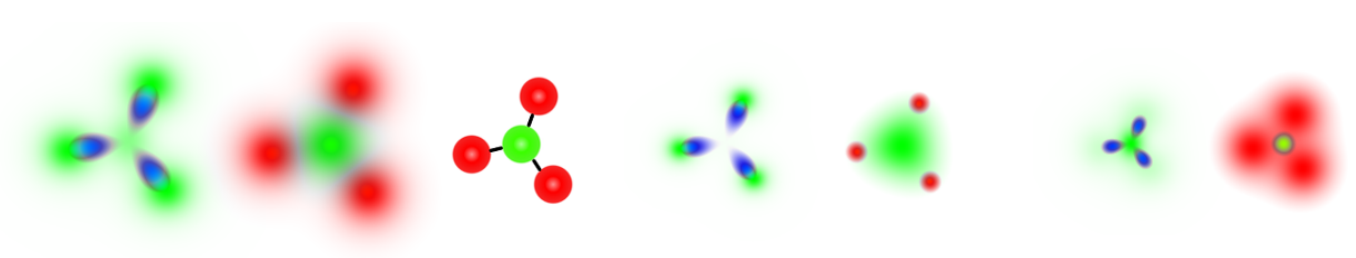

(b) $\left(Q_{1}, Q_{2}\right)=(3,1)$
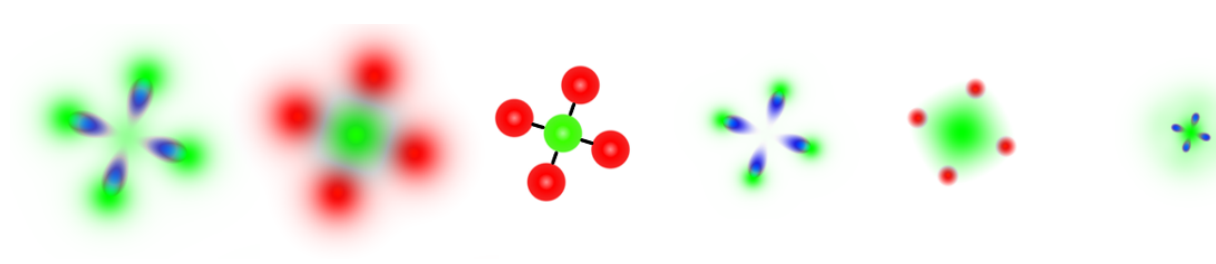

8

(c) $\left(Q_{1}, Q_{2}\right)=(4,1)$

Figure 16: $\left(Q_{1}, Q_{2}\right)=(Q, 1)$ minimal vortex "flowers", with $Q=2,3,4$. Each subfigure contains 7 elements from left to right: for column 1 through $3: v_{1} / v_{2}=1$ : an energy density plot, a field density plot and a sketch of how the red and green vortices are interconnected. For column 4 and 5: $v_{1} / v_{2}=2$ : an energy density plot and a field density plot. For column 6 and 7: $v_{1} / v_{2}=1 / 2$ : an energy density plot and a field density plot. All columns except column 3 are made using the coloring scheme of figure 2 .

the vortices and legs, the energy density with $v_{1} / v_{2}=2$, the field densities with $v_{1} / v_{2}=2$, the energy density with $v_{1} / v_{2}=1 / 2$ and finally the field densities with $v_{1} / v_{2}=1 / 2$.

Reiterating the properties of the vortex flowers for equal VEVs $\left(v_{1} / v_{2}=1\right)$ in figure 16 , we can see all vortex components in the energy density plots (first column) and the single bonds are clearly visible in blue as the contribution from the generalized Josephson term. From the field densities plot (second column), we can see that the vortices of both species have the same size.

We now turn to the larger VEV case for the red vortex (i.e. $v_{1} / v_{2}=2$ ), see the fourth and fifth columns in figure 16. First we notice that the energy density of the red vortices have shrunk and are locally so high that the energy density of the green vortex is barely visible. The single bonds are still visible, but have shrunk a bit due to the smaller size of the red vortices. The reason for the single bond length still being comparatively long is that the green vortex as the receptacle retains the same size as in the equal VEV case, thus setting the length scale between the small red vortices, viz. the petals. 

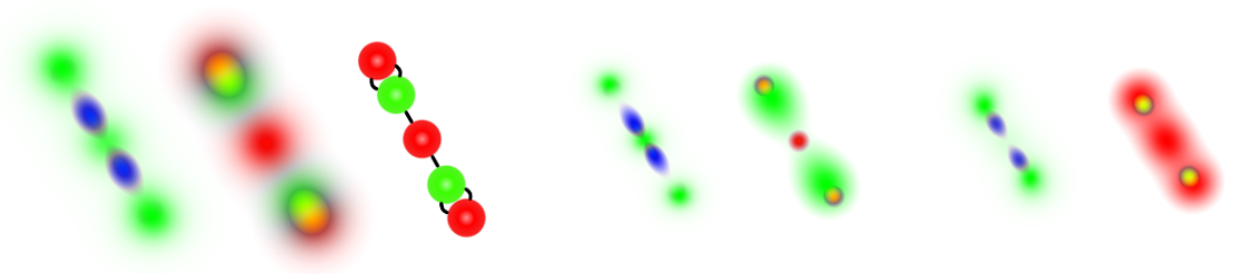

(a) $\left(Q_{1}, Q_{2}\right)=(3,2)$

Figure 17: $\left(Q_{1}, Q_{2}\right)=(Q+1, Q)$ vortex "stick", with $Q=2$. The figure contains 7 elements from left to right: for column 1 through 3: $v_{1} / v_{2}=1$ : an energy density plot, a field density plot and a sketch of how the red and green vortices are interconnected. For column 4 and 5: $v_{1} / v_{2}=2$ : an energy density plot and a field density plot. For column 6 and 7: $v_{1} / v_{2}=1 / 2$ : an energy density plot and a field density plot. All columns except column 3 are made using the coloring scheme of figure 2 .

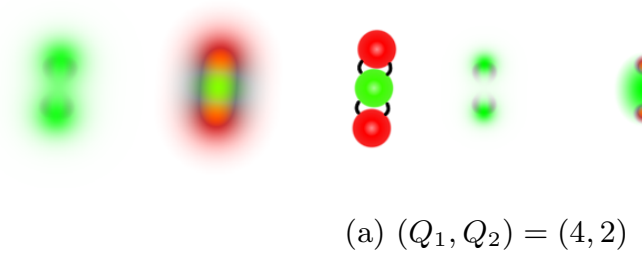

Figure 18: $\left(Q_{1}, Q_{2}\right)=(2 Q, Q)$ minimal vortex "pill", with $Q=2$. The figure contains 7 elements from left to right: for column 1 through $3: v_{1} / v_{2}=1$ : an energy density plot, a field density plot and a sketch of how the red and green vortices are interconnected. For column 4 and 5: $v_{1} / v_{2}=2$ : an energy density plot and a field density plot. For column 6 and 7: $v_{1} / v_{2}=1 / 2$ : an energy density plot and a field density plot. All columns except column 3 are made using the coloring scheme of figure 2 .

Turning now to the larger VEV case for the green vortex (i.e. $v_{1} / v_{2}=1 / 2$ ), see the sixth and seventh columns in figure 16 , we see a similar trend, but since it is now the receptacle that has shrunk and not the petals, the solutions are somewhat different in nature. First we notice that although the green vortex - the receptacle has shrunk significantly — the red vortices are still visible in the energy density plots, see the sixth column of the figure. Second, we notice that since the petals are forced to be closer to the receptacle and to one another, the single bonds binding the petals have been shortened significantly - in opposition to the $v_{1} / v_{2}=2$ case. Because the petals - the red vortices - are now closer to the receptacle and to one another, the $\left(Q_{1}, Q_{2}\right)=(4,1)$ vortex flower has almost lost its clear discrete $\mathbb{Z}_{Q_{1}}$ rotational symmetry (figure 16(c)), which however is still visible in the $\left(Q_{1}, Q_{2}\right)=(2,1)$ and $\left(Q_{1}, Q_{2}\right)=(3,1)$ solutions (figure $\left.16(\mathrm{a}, \mathrm{b})\right)$.

The last examples that we will display are the $\left(Q_{1}, Q_{2}\right)=(3,2)$ vortex "stick" and the $\left(Q_{1}, Q_{2}\right)=(4,2)$ vortex "pill", both possessing double bonds and the former also single bonds, see figures 17 and 18, respectively. 
Starting with the $\left(Q_{1}, Q_{2}\right)=(3,2)$ vortex "stick" of figure 17 , we again see that the energy density is localized around the three small red vortices in the fourth column of the figure, corresponding to the case of $v_{1} / v_{2}=2$; furthermore, the double bonds are tightly connected to the exterior red vortices and are visible in blue/gray representing the density of the generalized Josephson term. The single bonds, on the other hand, are not moved much and are strung over the length scale of the bond that is roughly set by the size of the green vortices, which are still being of normal size. In the other regime, i.e. $v_{1} / v_{2}=1 / 2$, the green vortices shrink and the red vortices are moved closer to one another, making the vortex "stick" a cloud of red vortex density with small concentrated green vortices chaining up the cloud, see the sixth and seventh columns of figure 17. In this case, the double bonds are barely visible, but the single bonds still are - although they have shrunk a bit too with respect to the $v_{1} / v_{2}=2$ case.

Finally, we come to the $\left(Q_{1}, Q_{2}\right)=(4,2)$ vortex "pill", which possesses only double bonds and is shown in figure 18. In the fourth and fifth columns, which correspond to the case of $v_{1} / v_{2}=2$, the red vortices are small satellites of the larger green vortex "cloud" and the double bonds are clearly visible in the fourth column of the figure. Somewhat surprisingly, the opposite case, $v_{1} / v_{2}=1 / 2$, which only shrinks the center green vortex of the "pill", shows a different behavior as the red vortices have collapsed into an indistinguishable cloud with a small green vortex in the center (shown with yellow color due to the overlap of vortex densities), see the seventh column of figure 18 .

\subsection{Global vortices}

In this section, we will consider an example of a global vortex. This is easily achieved and in fact there is a double infinity of global vortex solutions. The criteria for the global vortices is simply

$$
n_{\text {global }}=Q_{2} n_{1}-Q_{1} n_{2} \neq 0
$$

For concreteness, we consider the model with $\left(Q_{1}, Q_{2}\right)=(1,4)$ and place a single vortex in the first complex scalar field, i.e. $n_{1}=1$ and $n_{2}=0$, yielding

$$
n_{\text {global }}=4, \quad n_{\text {local }}=4,
$$

and the total winding number

$$
k=\frac{v_{1}^{2}}{v_{1}^{2}+16 v_{2}^{2}} .
$$

For simplicity, we will also consider only the equal VEV case, i.e. $v_{1}=v_{2}$, for which the winding number reduces to

$$
k=\frac{1}{17}
$$

Figure 19 shows a numerical calculation of the model with $\left(Q_{1}, Q_{2}\right)=(1,4)$ with a single vortex in the first scalar field, namely $n_{1}=1$ and $n_{2}=0$. Figure 19 (a) shows the 


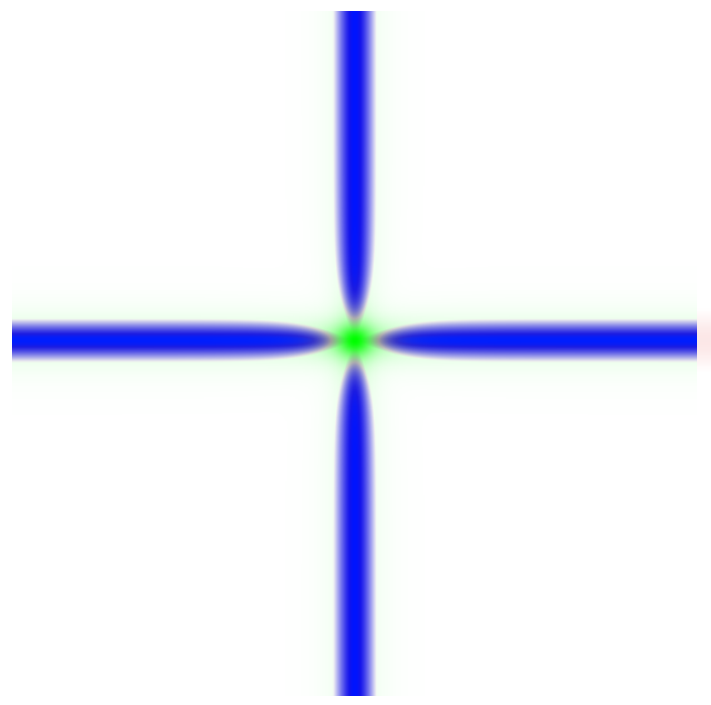

(a)

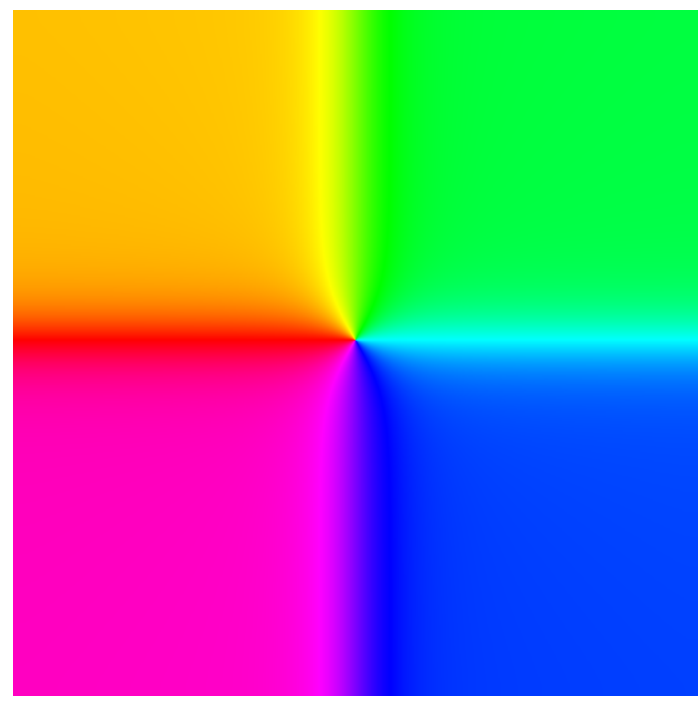

(c)

Figure 19: $\left(Q_{1}, Q_{2}\right)=(1,4)$ global vortex with four angular domain walls: (a) energy density (green) and density of the generalized Josephson term (blue) using the color scheme of figure 2(b); (b) field density using the color scheme of figure $2(\mathrm{a})$; (c) $\arg \left(\phi_{1}\right)$ mapped to the color wheel (hue).

energy density plot depicting the total energy density with green and the density of the generalized Josephson term with blue. Since the vortex is global as opposed to local, there are $Q_{2}=4$ domain walls emanating from the vortex core and extending towards spatial infinity. Figure 19(b) shows the field densities and only the red vortex field is visible; at the origin, the vortex is clearly seen as the scalar field has zero norm and hence deviates maximally from its VEV (from below) - this is marked with red color as opposed to the 


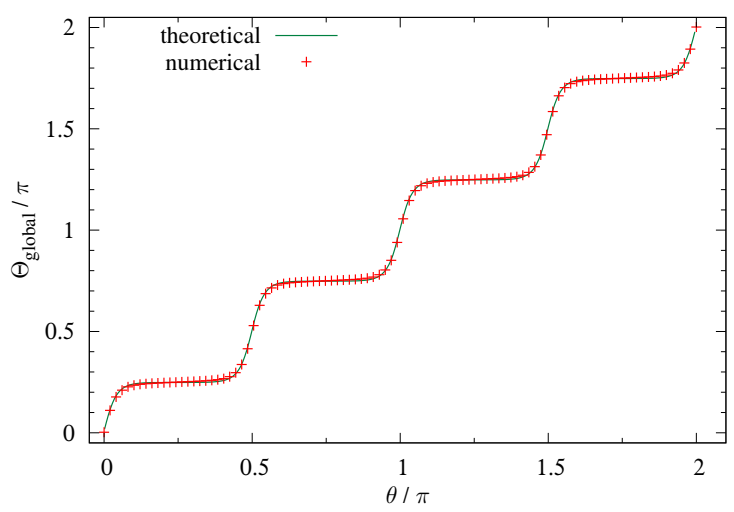

Figure 20: Numerical solution possessing four angular domain walls. The theoretical line in green is from eq. (2.51) and the red crosses are numerical phase data extracted from figure 19. The data are extracted at $r=9$ and here $\kappa=\sqrt{0.1\left(1+4^{2}\right)} \simeq 1.30384$ (see eq. (2.49)).

vacuum which is white. At the center of the domain wall (lines), the complex scalar field deviates slightly from its VEV and thus four dim red lines emanating from the vortex core are visible in figure 19(b). This is counter to the assumptions on which the analytic solution for the angular domain walls (2.51) were constructed. Finally, the phase of the complex scalar field, $\phi_{1}$, is shown in figure 19(c) with the argument of the complex scalar field mapped to the hue of the color wheel. With this convention $\arg \left(\phi_{1}\right)=0$ is red, $\arg \left(\phi_{1}\right)=2 \pi / 3$ is green, $\arg \left(\phi_{1}\right)=4 \pi / 3$ is blue, $\arg \left(\phi_{1}\right)=\pi / 3$ is yellow, $\arg \left(\phi_{1}\right)=\pi$ is cyan, and finally $\arg \left(\phi_{1}\right)=5 \pi / 3$ is magenta.

In figure 20 we show a comparison of the analytical prediction of the angular domain wall (2.51) and the numerically extracted phase data from figure 19. It is observed from figure 20, that the analytic solution works qualitatively very well and there is only a slight deviation from the numerical phase data near the centers of the domain wall. This deviation is caused by the assumption that the modulus of the complex scalar field, $\phi_{1}$, remains at its vacuum value $\left|\phi_{1}\right|=v_{1}$, which is only approximately true, see figure 19(b).

\section{Conclusion and discussion}

In this paper, we have studied a two-component Abelian-Higgs model with new cross interaction between the two complex scalar fields, which is inspired by the seminal Josephson term, but generalized to the case of the complex scalar fields carrying different electric charges. The new term is manifestly gauge invariant, but does not possess the physical interpretation of the interaction responsible for the Josephson effect, where quantum tunneling carries a Cooper pair across a thin layer to another superconductor.

After introducing the model and discussing the symmetries and the vacuum structure, we studied the vortices with and without the generalized Josephson term for generic charges, $\left(Q_{1}, Q_{2}\right)$. Our first result is that the total winding number $k$ (associated with the winding compensated by the gauge field $\left.A_{\theta}\right)$ is a rational fraction when $n_{\text {global }}=0$, but 
arbitrarily fractional when $n_{\text {global }} \neq 0$. Thereafter, we found the analytic angular domain wall solutions that exist in the generalized Josephson phase, which we denote the global vortex phase variable.

We considered also the conditions for having vortices with finite energy and found that for $\gamma=0$, corresponding to having turned off the generalized Josephson term, the total energy in the plane is logarithmically divergent with the cut-off scale (the largest length in the system) unless $n_{\text {global }}=0$, whereas for $\gamma \neq 0$ - i.e. with the generalized Josephson term taken into account - the total energy is linearly divergent with the cut-off scale, again unless $n_{\text {global }}=0$. The conclusion is thus that the only finite-energy vortices in the system, are local vortices (i.e. with $n_{\text {global }}=Q_{2} n_{1}-Q_{1} n_{2}=0$ ) - independently of whether the generalized Josephson term is turned on. The linearly divergent energy, of course, corresponds just to the fact that there are angular domain walls emanating from the global vortices and they tend to infinity or to the size of the superconducting system.

The numerical results of the paper comprise a full classification of the minimal local vortices, which have the smallest energies among their respective classes of electric charges. The classes of minimal local vortices contain a normal vortex, a vortex flower, a vortex stick, etc. The nonminimal local vortices exist in an infinite multitude and we have only provided a few interesting examples. Generally the nonminimal local vortex configurations appear in two categories: ring networks and stick networks - and both can be infinitely extended by trivial compositions.

Our final result is a comparison of the analytic angular domain wall solution with those possessed in a numerically found global vortex. The agreement is very good, even though the assumption of the complex scalar field to be at its VEV turns out not quite to hold true.

One improvement of the model would be the possibility to admit larger charges. If the charges have a common factor, a simple variant of the generalized Josephson term could be:

$$
-\gamma\left(\left(\phi_{1}^{Q_{2}} \bar{\phi}_{2}^{Q_{1}}\right)^{1 / \operatorname{gcd}\left(Q_{1}, Q_{2}\right)}+\left(\bar{\phi}_{1}^{Q_{2}} \phi_{2}^{Q_{1}}\right)^{1 / \operatorname{gcd}\left(Q_{1}, Q_{2}\right)}\right)
$$

which nevertheless does not ameliorate the situation of a large charge ratio. Obviously, the problem of having a power less than one of either of the fields in the generalized Josephson term leads to an equation of motion with a point singularity at the vortex positions.

The immediate generalization of our work is to consider $N$-component superconductors or $N$ flavors of complex scalar fields [8]. For $N=3$ flavors with equal charge, the minimal local vortex takes the form of a Y-shaped junction of three fractional vortices [39]. Global analogues of BECs with three or more components allow for molecules made of $N$ vortex species $[40,41]$. It would be interesting to study the details of the solutions for such multi-component systems with $N \geq 3$ and generic electric charges.

Conventional metallic superconductors with the order parameter (scalar field) with electric charge $2 e$ exhibit a $\mathbb{Z}_{2}$ topological order, due to nontrivial linking of a Wilson loop and a vortex, associated with the spontaneous symmetry breaking of a $\mathbb{Z}_{2}$ one-form symmetry [42]. It is an interesting question whether our model with generic charges exhibits a similar type of topological order. 
The more interesting development of this model would be to find a situation in condensed matter physics or other areas of physics, where the newly introduced term - the generalized Josephson term - is realized as a physical effect.

\section{Acknowledgments}

S. B. G. thanks Keisuke Ohashi and Calum Ross for discussions. C. C. is supported by JSPS KAKENHI Grant Number 19K14713. S. B. G. thanks the Outstanding Talent Program of Henan University for partial support. The work of S. B. G. is supported by the National Natural Science Foundation of China (Grant No. 11675223). M. N. is supported by the Ministry of Education, Culture, Sports, Science (MEXT)-Supported Program for the Strategic Research Foundation at Private Universities "Topological Science" (Grant No. S1511006). M. N. is also supported in part by JSPS KAKENHI Grant Numbers $16 \mathrm{H} 03984$ and $18 \mathrm{H} 01217$. M. N. is also supported in part by a Grant-in-Aid for Scientific Research on Innovative Areas "Topological Materials Science" (KAKENHI Grant No. 15H05855) from MEXT of Japan.

Open Access. This article is distributed under the terms of the Creative Commons Attribution License (CC-BY 4.0), which permits any use, distribution and reproduction in any medium, provided the original author(s) and source are credited.

\section{References}

[1] A.A. Abrikosov, On the Magnetic properties of superconductors of the second group, Sov. Phys. JETP 5 (1957) 1174 [Zh. Eksp. Teor. Fiz. 32 (1957) 1442] [INSPIRE].

[2] H.B. Nielsen and P. Olesen, Vortex Line Models for Dual Strings, Nucl. Phys. B 61 (1973) 45 [INSPIRE].

[3] A. Jaffe and C. Taubes, Progress in Physics. Vol. 2: Vortices and Monopoles - Structure of Static Gauge Theories, Birkhäuser, Boston U.S.A. (1980).

[4] E. Babaev, A. Sudbo and N.W. Ashcroft, A Superconductor to superfluid phase transition in liquid metallic hydrogen, Nature 431 (2004) 666 [cond-mat/0410408] [INSPIRE].

[5] E. Zohar, J.I. Cirac and B. Reznik, Simulating Compact Quantum Electrodynamics with ultracold atoms: Probing confinement and nonperturbative effects, Phys. Rev. Lett. 109 (2012) 125302 [arXiv:1204.6574] [INSPIRE].

[6] E.-G. Moon, Skyrmions with quadratic band touching fermions: A way to achieve charge $4 e$ superconductivity, Phys. Rev. B 85 (2012) 245123 [arXiv:1202.5389] [INSPIRE].

[7] J. Garaud and E. Babaev, Topological defects in mixtures of superconducting condensates with different charges, Phys. Rev. B 89 (2014) 214507 [arXiv: 1403.3373] [INSPIRE].

[8] E. Babaev, Phase diagram of a planar two band superconductor: Condensation of vortices with fractional flux quantum and existence of a nonsuperconducting superfluid state in this system, Nucl. Phys. B 686 (2004) 397 [cond-mat/0201547] [INSPIRE].

[9] E.V. Herland, E. Babaev and A. Sudbø, Phase transitions in a three dimensional $\mathrm{U}(1) \times \mathrm{U}(1)$ lattice London superconductor: Metallic superfluid and charge-4e superconducting states, Phys. Rev. B 82 (2010) 134511 [arXiv:1006.3311]. 
[10] B.D. Josephson, Possible new effects in superconductive tunnelling, Phys. Lett. 1 (1962) 251 [INSPIRE].

[11] B.D. Josephson, The discovery of tunnelling supercurrents, Rev. Mod. Phys. 46 (1974) 251 [INSPIRE].

[12] T.A. Fulton, P.L. Gammel, D.J. Bishop, L.N. Dunkleberger and G.J. Dolan, Observation of combined Josephson and charging effects in small tunnel junction circuits, Phys. Rev. Lett. 63 (1989) 1307.

[13] G. Wendin, Quantum information processing with superconducting circuits: a review, Rep. Prog. Phys. 80 (2017) 106001 [arXiv: 1610.02208].

[14] M. R. Matthews et al., Watching a Superfluid Untwist Itself: Recurrence of Rabi Oscillations in a Bose-Einstein Condensate, Phys. Rev. Lett. 83 (1999) 3358 [cond-mat/9906288].

[15] J. Williams, R. Walser, J. Cooper, E.A. Cornell and M. Holland, Excitation of a dipole topological state in a strongly coupled two-component Bose-Einstein condensate, Phys. Rev. A 61 (2000) 033612 [cond-mat/9904399].

[16] T. Zibold, E. Nicklas, C. Gross and M.K. Oberthaler, Classical Bifurcation at the Transition from Rabi to Josephson Dynamics, Phys. Rev. Lett. 105 (2010) 204101 [arXiv:1008.3057].

[17] M. Nitta, Josephson vortices and the Atiyah-Manton construction, Phys. Rev. D 86 (2012) 125004 [arXiv: 1207.6958] [INSPIRE].

[18] M. Kobayashi and M. Nitta, sine-Gordon kinks on a domain wall ring, Phys. Rev. D 87 (2013) 085003 [arXiv: 1302.0989] [INSPIRE].

[19] T. Fujimori, H. Iida and M. Nitta, Field theoretical model of multilayered Josephson junction and dynamics of Josephson vortices, Phys. Rev. B 94 (2016) 104504 [arXiv:1604.08103] [INSPIRE].

[20] M. Nitta, Josephson junction of non-Abelian superconductors and non-Abelian Josephson vortices, Nucl. Phys. B 899 (2015) 78 [arXiv:1502.02525] [INSPIRE].

[21] M. Nitta, Josephson instantons and Josephson monopoles in a non-Abelian Josephson junction, Phys. Rev. D 92 (2015) 045010 [arXiv:1503.02060] [INSPIRE].

[22] M. Nitta, Non-Abelian sine-Gordon Solitons, Nucl. Phys. B 895 (2015) 288 [arXiv: 1412.8276] [INSPIRE].

[23] G.C. Branco, P.M. Ferreira, L. Lavoura, M.N. Rebelo, M. Sher and J.P. Silva, Theory and phenomenology of two-Higgs-doublet models, Phys. Rept. 516 (2012) 1 [arXiv:1106.0034] [INSPIRE].

[24] M. Eto, M. Kurachi and M. Nitta, Constraints on two Higgs doublet models from domain walls, Phys. Lett. B 785 (2018) 447 [arXiv: 1803.04662] [INSPIRE].

[25] M. Eto, M. Kurachi and M. Nitta, Non-Abelian strings and domain walls in two Higgs doublet models, JHEP 08 (2018) 195 [arXiv:1805.07015] [INSPIRE].

[26] A.V. Ustinov, Solitons in Josephson junctions, Physica D 123 (1998) 315.

[27] Y. Tanaka, Phase Instability in Multi-band Superconductors, J. Phys. Soc. Jp. 70 (2001) 2844.

[28] Y. Tanaka, Soliton in Two-Band Superconductor, Phys. Rev. Lett. 88 (2001) 017002. 
[29] D.T. Son and M.A. Stephanov, Domain walls in two-component Bose-Einstein condensates, Phys. Rev. A 65 (2002) 063621 [cond-mat/0103451] [INSPIRE].

[30] E. Babaev, Vortices carrying an arbitrary fraction of magnetic flux quantum in two gap superconductors, Phys. Rev. Lett. 89 (2002) 067001 [cond-mat/0111192] [INSPIRE].

[31] K. Kasamatsu, M. Tsubota and M. Ueda, Vortex molecules in coherently coupled two-component Bose-Einstein condensates, Phys. Rev. Lett. 93 (2004) 250406 [cond-mat/0406150].

[32] J. Goryo, S. Soma and H. Matsukawa, Deconfinement of vortices with continuously variable fractions of the unit flux quanta in two-gap superconductors, Eur. Phys. Lett. 80 (2007) 17002 [cond-mat/0608015].

[33] M. Tylutki, L.P. Pitaevskii, A. Recati and S. Stringari, Confinement and precession of vortex pairs in coherently coupled Bose-Einstein condensates, Phys. Rev. A 93 (2016) 043623 [arXiv: 1601.03695] [INSPIRE].

[34] M. Eto and M. Nitta, Confinement of half-quantized vortices in coherently coupled Bose-Einstein condensates: Simulating quark confinement in a QCD-like theory, Phys. Rev. A 97 (2018) 023613 [arXiv: 1702.04892] [INSPIRE].

[35] M. Cipriani and M. Nitta, Crossover between integer and fractional vortex lattices in coherently coupled two-component Bose-Einstein condensates, Phys. Rev. Lett. 111 (2013) 170401 [arXiv: 1303.2592] [INSPIRE].

[36] M. Kobayashi, M. Eto and M. Nitta, Berezinskii-Kosterlitz-Thouless Transition of Two-Component Bose Mixtures with Intercomponent Josephson Coupling, Phys. Rev. Lett. 123 (2019) 075303 [arXiv:1802.08763] [INSPIRE].

[37] M. Kawasaki and K. Nakayama, Axions: Theory and Cosmological Role, Ann. Rev. Nucl. Part. Sci. 63 (2013) 69 [arXiv:1301.1123] [InSPIRE].

[38] M. Eto, Y. Hirono, M. Nitta and S. Yasui, Vortices and Other Topological Solitons in Dense Quark Matter, PTEP 2014 (2014) 012D01 [arXiv: 1308.1535] [INSPIRE].

[39] M. Nitta, M. Eto, T. Fujimori and K. Ohashi, Baryonic Bound State of Vortices in Multicomponent Superconductors, J. Phys. Soc. Jap. 81 (2012) 084711 [arXiv:1011.2552] [INSPIRE].

[40] M. Eto and M. Nitta, Vortex trimer in three-component Bose-Einstein condensates, Phys. Rev. A 85 (2012) 053645 [arXiv: 1201.0343] [InSPIRE].

[41] M. Eto and M. Nitta, Vortex graphs as $N$-omers and $C P(N-1)$ Skyrmions in $N$-component Bose-Einstein condensates, EPL 103 (2013) 60006 [arXiv:1303.6048] [INSPIRE].

[42] T.H. Hansson, V. Oganesyan and S.L. Sondhi, Superconductors are topologically ordered, Annals Phys. 313 (2004) 497 [cond-mat/0404327]. 